This PDF is a selection from an out-of-print volume from the National Bureau of Economic Research

Volume Title: Seasonal Analysis of Economic Time Series

Volume Author/Editor: Arnold Zellner

Volume Publisher: NBER

Volume URL: http://www.nber.org/books/zell78-1

Publication Date: 1978

Chapter Title: A Time Series Analysis of Seasonality in Econometric Models

Chapter Author: Charles I. Plosser

Chapter URL: http://www.nber.org/chapters/c4331

Chapter pages in book: (p. 365 - 408) 


\title{
A TIME SERIES ANALYSIS OF SEASONALITY IN ECONOMETRIC MODELS
}

\author{
Charles I. Plosser \\ Stanford University
}

\section{INTRODUCTION}

The traditional literature on seasonality has mainly focused attention on various statistical procedures for obtaining a seasonally adjusted time series from an observed time series that exhibits seasonal variation. Many of these procedures rely on the notion that an observed time series can be meaningfully divided into several unobserved components. Usually, these components are taken to be a trend or cyclical component, a seasonal component, and an irregular or random component. Unfortunately, this simple specification, in itself, is not sufficient to identify a unique seasonal component, given an observed series. Consequently, there are difficult problems facing those wishing to obtain a seasonally adjusted series. For example, the econometrician or statistician involved in this adjusting process is immediately confronted with several issues. Are the components additive or multiplicative? Are they deterministic or stochastic? Are they independent or are there interaction effects? Are they stable through time or do they vary through time? Either explicitly or implicitly, these types of questions must be dealt with before one can obtain a seasonally adjusted series.

One approach to answering some of these questions would be to incorporate subject matter considerations into the decision process. In particular, economic concepts may be useful in arriving at a better understanding of seasonality. Within the context of an economic structure (e.g., a simple supply and demand model), the seasonal variation in one set of variables, or in one market, should have implications for the seasonal variation in closely related variables and markets. ${ }^{1}$ For example, the seasonality in the amount of labor supplied in nonagricultural

\footnotetext{
1 Kuznets [11] was concerned with how seasonal movements worked their way through various markets. Fundamental to this approach is the idea of induced or derived seasonal variation. That is, seasonality is induced into some markets because of seasonality in other markets. However, Kuznets first obtained what he called the seasonal component of an observed series and proceeded to compare these seasonal components in related markets.

${ }^{2}$ An example of how an economic model can be built to generate seasonal or periodic behavior can be found in Crutchfield and Zellner [22].

${ }^{3}$ Laffer and Ranson [12] were concerned with this problem and made use of seasonal dummies in an attempt to avoid the dependence on the seasonally adjusted data.
}

labor markets is not independent of the labor demanded in agricultural labor markets. Consequently, knowledge of the economic structure can provide one with a great deal of understanding about the seasonal variation of different variables, such as where it comes from and what might cause it to vary through time.

The purpose of this paper is to suggest and investigate an approach that involves the incorporation of seasonality directly into an economic model. ${ }^{2}$ Analyzing the problem from this perspective has two important implications. First, if an adjusted series is the objective, an economic model that incorporates seasonality may provide an analyst with a better understanding of the source and type of seasonal variation, as indicated in the previous paragraph. This understanding, in turn, may aid in the development of improved adjustment procedures. Second, including seasonality in an economic model avoids the necessity of using a seasonally adjusted data base in estimating an economic model and subsequent concern over whether the seasonal adjustment procedure itself may be causing distortions of the economic analysis and the interpretation of the model. ${ }^{3}$ For example, although many economic time series are available in adjusted form, there are some series that are not adjusted at all (e.g., interest rates). Wallis [23] shows how the use of adjusted and unadjusted data in the same model can lead to spurious dynamic relationships between variables where dynamic relationships do not otherwise exist.

Furthermore, to the extent model builders do not take seasonality into account in the specification of a model because they believe that using seasonally adjusted data has eliminated that need, they could be led into model

Charles 1. Plosser is an assistant professor at the Graduate School of Business, Stanford University, Stanford, Calif. This work has been financed, in part, by the National Science Foundation under Grant GS 40033 and the H. G. B. Alexander Research Foundation, Graduate School of Business, University of Chicago. The author is grateful to Arnold Zellner for his helpful comments and encouragement. J. M. Abowd, R. E. Lucas, H. V. Roberts, G.W. Schwert, and W.E. Wecker also provided helpful suggestions. All remaining errors, however, remain the sole responsibility of the author. 
misspecification, misleading inferences about parameter values, and poor forecasts. Such problems would naturally arise if the adjustment procedure did not effectively eliminate the seasonal variation in the data. Consequently, the adjustment procedure may have the effect of inducing properties on a series that are spurious concerning the model under consideration.

In Zellner and Palm [26], techniques were developed for analyzing dynamic econometric models that combined traditional econometric modeling with the time series techniques developed by Box and Jenkins [3]. In a subsequent work, Zellner and Palm [27], these techniques were applied to the analysis of several monetary models of the U.S. economy. Using monthly data for 1953-72, the information in the data was checked against the implications derived from these models. They pointed out, in the conclusion of their work, that even though they were using seasonally adjusted data, effects of seasonality seemed to be present in the autocorrelation structure of some of the variables, as well as in the residuals of the transfer functions. These complications might be expected from data that are smoothed in the same manner, regardless of the underlying stochastic process or economic mechanism at work.

Finally, if the data being used to test and estimate a model are inappropriate for the particular model, the model is likely to produce poor forecasts. Even in the case of forecasting univariate time series, the effects of seasonal adjustment may cause poor predictions. This lack of prediction accuracy may arise from the fact that the adjustment procedures periodically undergo revision, such that the form of the filter and the weights employed are changing through time. That is, the raw data are being passed through a filter that may vary considerably over a particular sample period. The result would be to introduce an instability in the stochastic properties of the adjusted data that may not exist in the raw or unadjusted data.

Figure 1 and 2 provide an illustrative example of the type of prediction problem suggested in the preceding paragraph. Using the methodology of Box and Jenkins [3], a univariate time series model was built for the unadjusted money stock (Ml). The model was identified using monthly data for January 1953-December 1962 and then used to forecast unadjusted M1 through 1963 (i.e., forecasting up to 12 steps ahead). Subsequently, the model was updated with actual data through December 1963 and then used to forecast M1 for 1964. This process was repeated through 1972 . The results of this exercise are presented graphically in figure 1. These are the plots of the actual and the predicted series as well as a set of 95 percent prediction intervals. As can be seen, the model seems to do rather well with the actual series coming close to being outside the prediction interval in 1967 and again in 1969. Even at the 12-step-ahead forecast, the error is rarely more than 1 to 2 percent.

In contrast to this is a model developed using the same techniques for the seasonally adjusted money supply. The same updating and prediction procedure was performed with the model, and the results are shown in figure 2 . Notice the relatively larger prediction errors at the 12-stepahead forecast. More important is the observation that the actual series often wanders outside the prediction interval. It is, of course, very difficult to compare the results in figures 1 and 2 directly, because, in fact, the models are predicting two different series. A complete analysis of the findings presented in figures 1 and 2 would constitute a study in and of itself, but such an analysis is not the intent of this work. However, these simple results should be sufficient to cause one to ask questions concern. ing the role of adjustment, and, perhaps, its usefulness in forecasting. ${ }^{4}$

The organization of the remainder of this paper is as follows: The second section is a methodological section that includes a brief discussion of the analysis of linear dynamic econometric models as developed in Zellner [25] and Zellner and Palm [26], as well as some of the theoretical aspects involved in modeling seasonal time series. Suggestions are then made concerning the way one might go about building seasonality into a model and how to check the consistency of the specification of the model with data. In the third section, a simple economic model is proposed with explicit assumptions regarding the manner in which seasonality enters the system. This is followed by a detailed discussion of the implications of the model for the properties of the stochastic processes for the endogenous variables. In particular, consideration is given to how the effects of changes in the values of structural parameters and of properties of the processes for exogenous variables that would lead to changes in the seasonal properties of the output variables of the model. The fourth section presents the results of an empirical analysis of the model, and the fifth section provides a discussion of the results and implications for future research.

\section{METHODOLOGY FOR ANALYZING SEASONAL ECONOMIC MODELS}

In this section, methodology is suggested for analyzing seasonal economic models. In the subsection on the analysis of linear dynamic econometric models, a brief discussion is provided of the analysis of linear dynamic econometric models as developed by Zellner [25] and Zellner and Palm [26]. In the subsection on seasonality in time series data, several approaches to modeling data with seasonal variation are discussed. Finally, in the subsection on an approach to the analysis of seasonality in structural models, the methodology developed in the subsection on the analysis of linear dynamic econometric models and the subsection on seasonality of time series

\footnotetext{
- There are certainly alternative explanations for this observed phenomenon. However, these results are only meant to be suggestive, and not conclusive evidence of the distortions that may be caused by seasonal adjustment. The reader who is interested in the details of the development of the exact models used for this example are referred to Plosser [17]
} 
(In billions of dollars)

Model $(0,1,3)(0,1,1)_{12}$
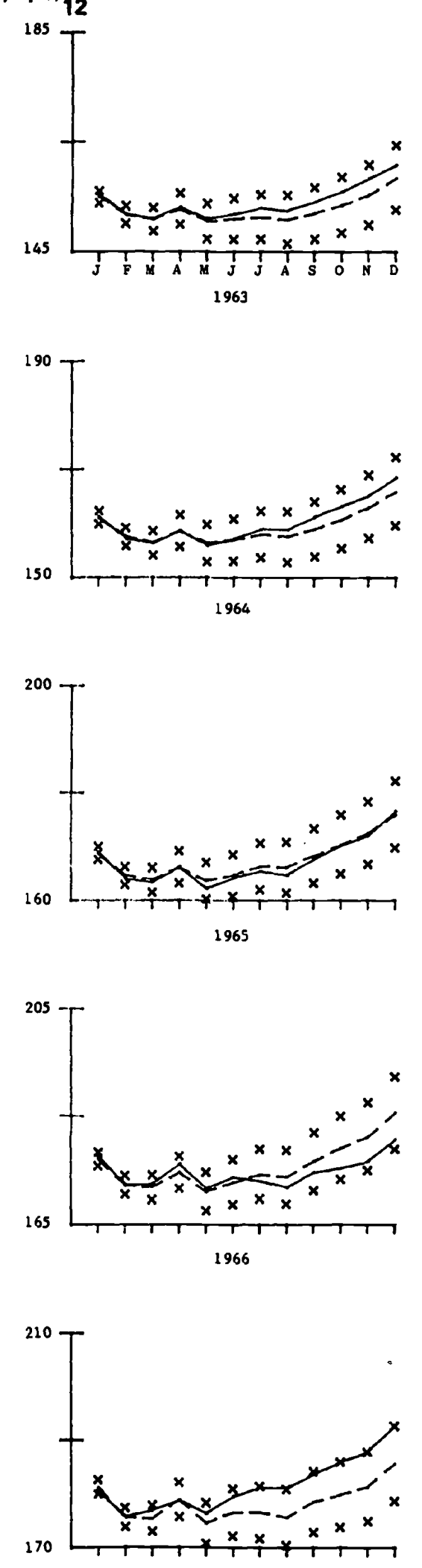

Approximate 95-percent prediction interval.
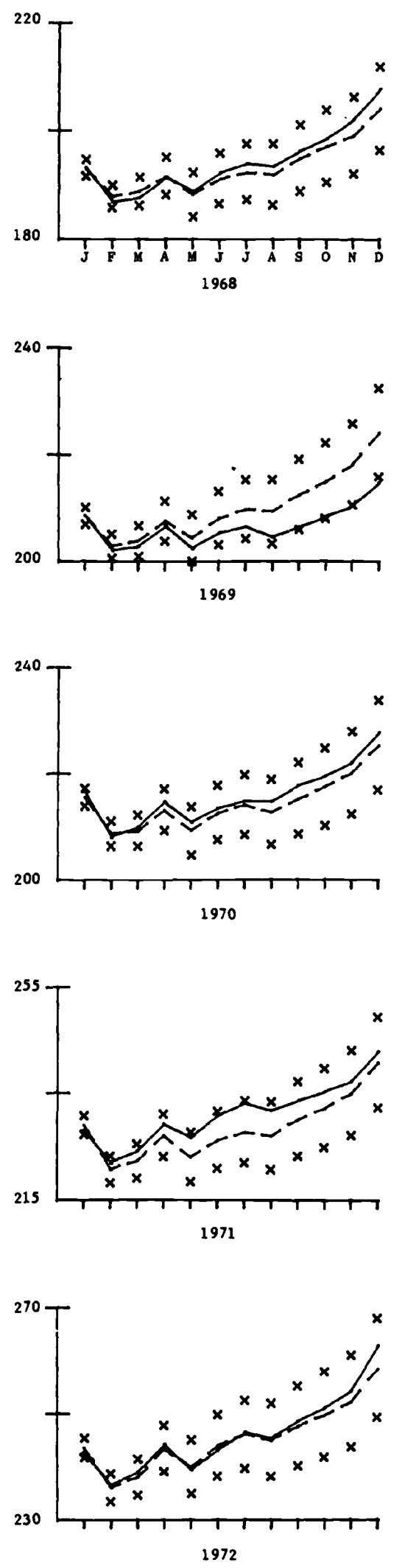
Figure 2. UPDATED PREDICTIONS FOR M1, ADJUSTED

(In billions of dollars)

Modal $(0,1,3)$
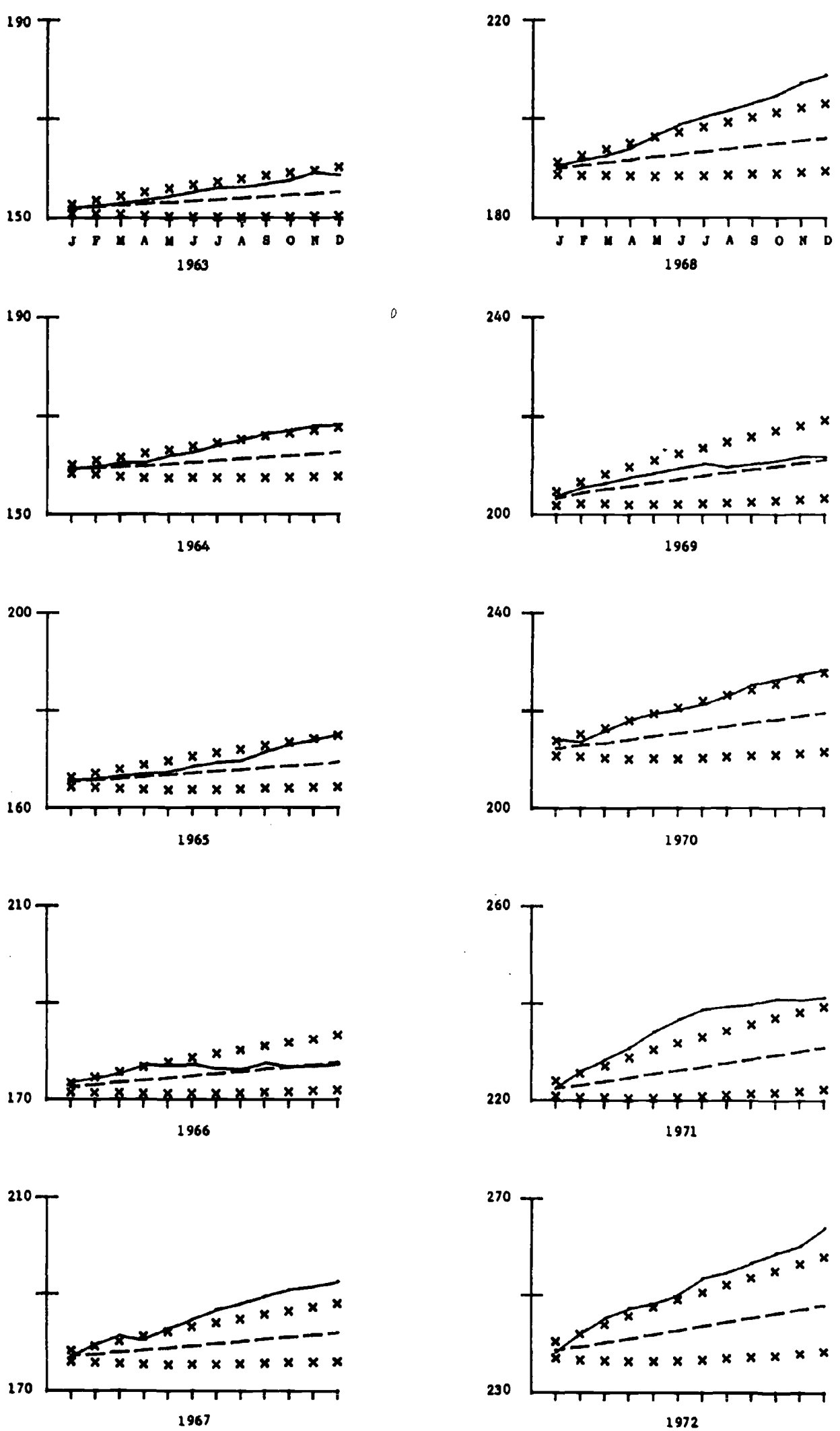

-

- - For ocest.

- Approximate 95-percent prediction interval.
PLOS

data

seasc

chect

the $d$

Anal:
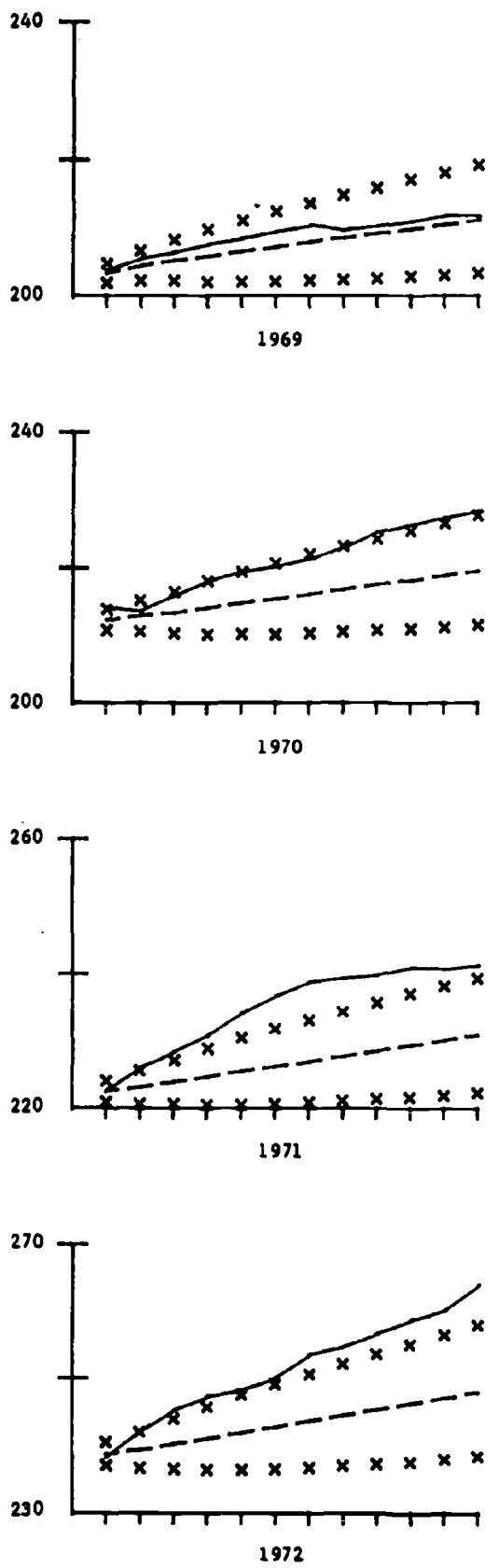

whe] writt

pxl

oper

matı

poly

assu

whe

mat'

$\mathrm{T}$

simı

that

sugg

bein

syste

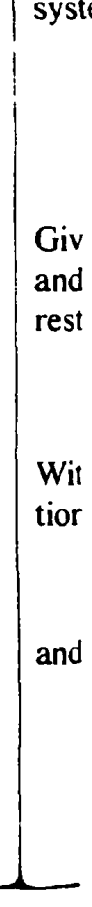


data is utilized to illustrate several ways of incorporating seasonality in an econometric model and techniques for checking the model's specification against information in the data.

\section{Analysis of Linear Dynamic Econometric Models}

As indicated by Quenouille [19] and Zellner and Palm [26], a linear multiple time series (MTS) process can be written as follows:

$$
\begin{aligned}
& H(L) \underline{z}_{t}=F(L) \underline{e}_{t}, \\
& \text { for } t=1,2, \ldots, T \\
& \operatorname{pxp} p x 1 \quad p x p p x 1
\end{aligned}
$$

where $z_{t}$ is a vector of $p$ observable variables (in this case written as deviations from their respective means), $e_{l}$ is a $p x 1$ vector of unobservable random errors, $L$ is the lag operator such that $L^{k} x_{t}=x_{t-k}$ and $H(L)$ and $F(L)$ are pxp matrices of full rank having elements that are finite polynomials in $L$. In addition, the error vector $\underline{e}_{t}$ is assumed to have the following properties:

$$
\begin{gathered}
E \underline{e}_{t}=\underline{0} \\
\text { for all } t, t^{*} \\
E \underline{e}_{t} \underline{e}_{t}^{\prime}=\delta_{t t^{*}} I_{p}
\end{gathered}
$$

where $\delta_{t t^{*}}$ is the Kronecker delta and $I_{p}$ is a pxp-unit matrix. Note that contemporaneous and serial correlations between errors are introduced through $F(L$ ).

This general MTS model includes the line:ar dynamic simultaneous equation model as a special case. Assume that prior information, in particular economic theory, suggests that certain elements of $z_{t}$ can be treated as being endogenous and others as being exogenous. The system (1) can then be written as follows:

$$
\left[\begin{array}{ll}
H_{11} & H_{12} \\
H_{21} & H_{22}
\end{array}\right]\left[\begin{array}{l}
\underline{y}_{t} \\
\underline{x}_{t}
\end{array}\right]=\left[\begin{array}{ll}
F_{11} & F_{12} \\
F_{21} & F_{22}
\end{array}\right]\left[\begin{array}{l}
\underline{e}_{1 t} \\
\underline{e}_{2 t}
\end{array}\right]
$$

Given that $y_{t}$ represents a vector of endogenous variables and $x_{t}$ a vector of exogenous variables, the following restrictions are implied:

$$
H_{21} \equiv 0, F_{12} \equiv 0 \text { and } F_{21} \equiv 0
$$

With these restrictions imposed, the usual structural equations from (3) are given in (5):

$$
H_{11} \underline{y}_{t}+H_{12} \underline{x}_{t}=F_{11} \underline{e}_{1 t}
$$

and

$$
H_{22} \underline{x}_{t}=F_{22} \underline{e}_{2 t}
$$

represents an autoregressive moving average process generating the exogenous variables. ${ }^{5}$

If it is now assumed that the roots of the determinantal equations $\left|H_{11}(\xi)\right|=0$ and $\left|H_{22}(\xi)\right|=0$ lie outside the unit circle, the system (5) can be rewritten in two forms that can be of 'ise in analyzing the model. The first form represents a system of final equations (FE's) for the endogenous variables. They are obtained by substituting for $x_{t}$ in (5) the expression

$$
\underline{x}_{t}=H_{22}^{-1} F_{22} \underline{e}_{2 t}
$$

and then premultiplying both sides of the resulting expression by the adjoint of $H_{11}$ that yields

$$
\mid H_{11} \underline{y}_{t}+H_{11}^{*} H_{12} H_{22}^{-1} F_{22} \underline{e}_{2 t}=H_{11}^{*} F_{11} \underline{e}_{1 t}
$$

or

$$
\left|H_{11}\right|\left|H_{22} \underline{y}_{t}=-H_{11}^{*} H_{12} H_{22}^{*} F_{22} e_{2 t}+\right| H_{22} \mid H_{11}^{*} F_{11} e_{1 t}
$$

where $\left|H_{v}\right|$ denotes the determinant and $H_{v}^{*}$ the adjoint matrix of $H_{i j}$. This representation implies that each endogenous variable can be written in the form of an autoregressive-integrated moving average (ARIMA) model of the type developed and analyzed by Box and Jenkins [3]. Thus, as emphasized by Zellner and Palm, those who utilize the Box and Jenkins models for forecasting are not making use of a technique that is necessarily distinct from standard econometric models. In fact, they are utilizing a very specialized reduced form, the FE, that is well suited for forecasting but may or may not be very informative for structural analysis. However, this representation of the model can provide insights into the stochastic structure of the endogenous variables in the system. For example, if one is interested in seasonality, the autocorrelation coefficient at the seasonal lag can be analyzed with respect to changes in structural parameters or changes in the processes generating the exogenous variables. Furthermore, this type of analysis is helpful in understanding what type of adjustment procedure may be suggested by the model.

Upon inspection, several things can be noted about (9). First, since the assumption is made that all the elements of $F(L)$ and $H(L)$ are finite polynomials in $L$, then if no cancellation takes place, it is apparent that each and every endogenous variable in the system will have identical autoregressive (AR) polynomials and they will be of order equal to or greater than the AR polynomials for the elements of $\underline{x}_{t}$. This theoretical restriction might be one means of testing the model against information obtained from the data. In addition, there are restrictions placed on the form of the moving average (MA) polynomial in (9). However, there are possible reasons why these theoretical restrictions on the AR and MA polynomials may not be observed in the data even when the model is true. One

\footnotetext{
II one or more of the elements of $x$, is deterministic, it can not be handled in this fashion but must be analyzed through the transfer functions, a discussion of which will follow.
} 
problem, mentioned by Zellner and Palm, is the possibility of cancellation. This will occur if there are common roots in the AR and MA portions. Depending upon the complexity of the structural model, this may or may not be noticed by the analyst but if not recognized could lead to estimated FE's that do not appear to satisfy the restrictions implied on the polynomials by the model. ${ }^{6}$

The second set of equations derived from the system (5) that can be of value in testing assumptions about the structural model is the set of transfer functions (TF's). These equations can be obtained from (5) by multiplying both sides by $H_{11}^{*}$; this yields

$$
\left|H_{11}\right| \underline{y}_{t}=-H_{11}^{*} H_{12} \underline{x}_{t}+H_{11}^{*} F_{11} e_{1 t}
$$

or, alternatively,

$$
y_{t}=\frac{-H_{11}^{*} H_{12}}{\left|H_{11}\right|} x_{t}+\frac{H_{11}^{*} F_{11}}{\left|H_{11}\right|} \underline{e}_{1 t}
$$

As noted by Zellner and Palm [26], Pierce and Mason [16], and Kmenta [10], this form expresses the current values of endogenous variables as functions of the current and past values of the exogenous variables and is restricted in form. Formally, (11) is a set of rational distributed lag (RDL) equations, Jorgenson [9] and Dhrymes [6], or a system of multiple-input transfer functions (MITF) of the type described by Box and Jenkins [3].

This form of the model is useful for prediction and control. In particular, it is useful for assessing the response, over time and in total, of endogenous variables to changes in exogenous variables. Notice that, here too, there are strong restrictions on the form of the TF's under the assumptions of a specific model. For instance, if no cancellation occurs and if all the elements of $H_{11}^{*}$ and $H_{12}$ are finite polynomials, then all of the inputs have the same denominator polynomial. There are also restrictions on the form of the error process in (11). Other tests that could be carried out concern testing the assumptions of the exogeneity of the $x_{\imath}$ 's. By estimating and analyzing (11) and comparing the results with the restrictions implied by a specific structural model, it is felt that many useful insights can be obtained concerning the adequacy of the specification of the structure. In particular, interest here will focus on the specification of the seasonal aspects of the model.

\section{Seasonality in Time Series Data}

Before discussing how one would incorporate seasonality in a structural model, it will be useful to review briefly several approaches to modeling data that have seasonal properties. The two approaches discussed here are the traditional concept of seasonality that treats an observed series as the sum of three components, a trend or cyclical

\footnotetext{
${ }^{6}$ Of course, if the model is incorrect or misspecified, then these restrictions will also fail to hold.
}

component, a seasonal component and a noise companent, ${ }^{7}$ and the multiplicative times series model as devel. oped by Box and Jenkins [3].

One of the more common approaches to seasonality within the framework of the aforementioned traditional model is the dummy variable model. The general form of such a model is

$$
y_{t}=y_{t}+\sum_{i=1}^{s} \alpha_{t} d_{\mathfrak{t}}+\epsilon_{t}
$$

where $y_{i}$ is the trend or cyclical component, $\epsilon_{t}$ is an error term, and the dummy variables $d_{i t}$ are used to represent the seasonal component of the series. (Oftentimes, $y_{i}^{c}$ is represented by a polynomial in $t$, time.) If monthly data were under consideration, one might use a dummy variable for each month representing a series with a fixed periodic or seasonal component. The estimate of $\alpha_{i}$ would represent the estimated mean for the $i^{\text {th }}$ month. If such a system is presumed to be the true model, it is then straightforward to obtain a seasonally adjusted series by just subtracting the seasonal component that yields

$$
y_{t}^{a}=y_{t}-y_{t}^{s}=y_{i}^{c}+\epsilon_{t}
$$

where

$$
y_{t}^{8}=\sum_{i=1}^{8} \alpha_{i} d_{i t}
$$

Another approach, also using this traditional decomposition, is the Census Bureau $X-11$ program. (See [21].) The basic idea of this approach is to eliminate the seasonal component $y_{t}^{s}$ through the application of symmetric moving average filters. That is, a seasonally adseries is obtained by passing the unadjusted data through a filter of the form

$$
y_{t}^{a}=\sum_{i=-k}^{k} \beta_{i} y_{t-i}=B(L) y_{t}
$$

where the $\beta_{i}$ 's are fixed weights such that $\beta_{i}=\beta_{-i}$ and $\sum_{i=-k}^{k} \beta_{i}=1$, and $L$ is the lag operator. In terms of the traditional components model, this filter is chosen such that the seasonal component is taken out and the trend or cyclical component is unaffected. (That is, $B(L) y_{i}^{c}=y_{i}^{c}$, and $B(L) y_{i}^{g}=0$.)

Another class of models that is of special interest and that contains the dummy variable approach as a special case, is the multiplicative seasonal time series models of Box and Jenkins. These models are of the general form:

$$
\Gamma_{P}\left(L^{s}\right) \phi_{p}(L) \Delta_{s}^{D} \Delta^{d} z_{t}=\Omega_{Q}\left(L^{s}\right) \theta_{q}(L) a_{t}
$$

\footnotetext{
'As noted earlier, it is in this conceptual framework that the idea that a series can be decomposed into a seasonal component and a seasonally adjusted series arises.
}

the

root

circ

$(p, a$

ple.

\section{Let}

diff

pro

pol

\section{of}

\section{tic}

\section{an}

\section{sC}

di

\section{di}


where $s$ is the length of the seasonal period (e.g., 12 for monthly data), $\Delta_{s}^{D}=\left(1-L^{s}\right)^{D}, \Delta^{d}=(1-L)^{d}, \Gamma$ and $\Omega$ are seasonal polynomials in $L^{s}$ of degree $P$ and $Q$ respectively, $\phi$ and $\theta$ are polynomials in $L$ of degree $p$ and $q$, respectively, and $a_{t}$ is a white-noise error term. It is also assumed that the roots of $\Gamma(\xi)=0$ and $\phi(\xi)=0$ lie outside the unit circle so that the process is stationary and the roots of $\Omega(\xi)=0$ and $\theta(\xi)=0$ lie on or outside the unit circle. Box and Jenkins refer to this as a model of order $(p, d, q)(P, D, Q)_{s}$.

Consider the process $(0,1,1)(0,1,1)_{12}$ as a simple example. It can be written as

$$
\left(1-L^{12}\right)(1-L) z_{t}=\left(1-\theta_{1} L\right)\left(1-\Omega_{1} L^{12}\right) a_{t}
$$

Let $w_{t}=(1-L)\left(1-L^{12}\right) z_{t}$, i.e., let $w_{t}$ equal the seasonal differences of the changes in $z_{t}$. Now the moving average process governing $w_{t}$ is easily seen by multiplying out the polynomials on the right-hand side of (17), yielding

$$
w_{t}=\left(1-\theta_{1} L-\Omega_{1} L^{12}+\theta_{1} \Omega_{1} L^{13}\right) a_{t}
$$

Therefore, this multiplicative model can be interpreted as an ordinary MA process of order 13. The distinction is that the multiplicative formulation restricts the weights on lags 2 through 11 to be 0 and on lag 13 to be the product of the weights for lags 1 and 12 .

In general, these multiplicative seasonal models cannot be decomposed or interpreted within the traditional unobserved components framework without precise definitions of the components and some further identifying restrictions. ${ }^{8}$ However, there is one special case of (16) that has an interpretation as the dummy variable case described earlier. Assume that observations were taken quarterly on some variable $z_{t}$. In addition, assume that the true process generating the $z_{i}$ 's were such that each quarter had a different mean, but, otherwise, the series was just a random, nonautocorrelated variable, $a_{t}$. Such a process could be written as

$$
z_{t}=\alpha_{1} d_{1 t}+\alpha_{2} d_{2 t}+\alpha_{3} d_{3 t}+\alpha_{4} d_{4 t}+a_{t}
$$

where $d_{i t}$ is a dummy variable that takes on the value 1 in the $i^{\text {th }}$ quarter and 0 elsewhere. The estimates of the $\alpha_{i}$ 's would represent the mean of the $i^{\text {ih }}$ quarter. If one were to seasonally difference this process, then the remaining process would be

$$
\left(1-L^{4}\right) z_{t}=\left(1-L^{4}\right) a_{t}
$$

The effect of seasonal differencing is to eliminate a constant, deterministic seasonal pattern. The process in

\footnotetext{
${ }^{8}$ See Cleveland [5]. He has proposed an underlying stochastic process for which the Census $\mathrm{X}-11$ is nearly optimal from the standpoint of conditional expectation. He argues that, for processes very near this, the $X-11$ does quite well, but, when departures occur, the appropriateness of the $\mathrm{X}-11$ decomposition is thrown into doubt.
}

(20) indicates that under the particular model in (19), the seasonal differences of $z_{t}$ obey a first-order seasonal moving average process with a parameter value of 1 . Alternatively, if the $a$ 's were considered nonautocorrelated and the model were found to have a first-order seasonal moving average parameter of less than 1 , then the implication would be that the seasonal pattern is changing through time. That is, the seasonal means are changing through time. ${ }^{9}$

The multiplicative model will be used in this work because of its flexibility in describing not only certain types of additive or deterministic seasonal patterns but also seasonal patterns that might not be constant through time. In addition, it readily fits into the framework of analysis of this paper.

\section{An Approach to the Analysis of Seasonality in Structural Models}

One question with which this work is concerned is how seasonality enters a structural econometric model. The primary focus is on testing the assumption that seasonality enters the system through exogenous forces. That is, can the seasonal fluctuations of the endogenous variables of the system be explained by the seasonality of the exogenous variables? There are, of course, other possibilities, such as certain parameters in the structure that fluctuate seasonally and, therefore, induce a seasonal pattern in the endogenous variables even when the exogenous variables are nonseasonal.

One approach that might be put forward combines the traditional concept of seasonality and seasonal adjustment with the concepts and methodology presented in the subsection on the analysis of linear dynamic econometric models. Assume that the endogenous variables of the system, denoted by $y_{t}$, and the exogenous variables of the system, denoted by $x_{l}$, can be written as follows:

$$
\begin{aligned}
& \underline{y}_{t}=\underline{y}_{t}^{c}+\underline{y}_{t}^{s}+\underline{v}_{t} \\
& \underline{x}_{t}=\underline{x}_{t}^{c}+\underline{x}_{t}^{s}+\underline{u}_{t}
\end{aligned}
$$

where no superscript on $x$ or $y$ indicates an observed variable, a superscript $c$ denotes the trend or cyclical component, $s$ denotes the seasonal component, and $y_{t}$ and $\underline{u}_{t}$ are noise components. In addition, assume that one believes the true economic relationship is in terms of the trend components. In the notation of the subsection on the analyses of linear dynamic econometric models, the model can be written as

$$
H_{11} \underline{y}_{i}^{c}+H_{12} \underline{x}_{i}^{c}=F_{11} \underline{e}_{11}
$$

\footnotetext{
OThese models are the first satisfactory models for forecasting seasonal series with changing seasonal patterns. For a more complete development and discussion of these models see Box and Jenkins [3, ch. 9].
} 
Substitution yields

$$
H_{11}\left(\underline{y}_{t}-\underline{y}_{t} t-\underline{y}_{t}\right)+H_{12}\left(\underline{x}_{t}-\underline{x}_{l}^{8}-\underline{u}_{t}\right)=F_{11} \underline{e}_{1 t}
$$

or

$$
\begin{gathered}
\underline{y}_{t}=\underline{y}_{t}^{*}-\frac{H_{11}^{*} H_{12}}{\left|H_{11}\right|} \underline{x}_{t}+\frac{H_{11}^{*} H_{12}}{\left|H_{11}\right|} \underline{x}_{t}^{*} \\
+\frac{H_{11}^{*} F_{11}}{\left|H_{11}\right|} \underline{e}_{1 t}+\frac{H_{11}^{*} H_{12}}{\left|H_{11}\right|} \underline{u}_{t}+\underline{v}_{t}
\end{gathered}
$$

It is clear that if (22) is the true model, then the model builder must be very concerned about how the trend component is obtained from the observed or unadjusted data. On the other hand, such a theory could be tested using the unadjusted data and the seasonal components, using (24). For example, a restriction implied by (22) on (24) is that the coefficient of $y_{t}^{8}$ is 1 and the coefficient of $\underline{x}_{t}^{s}$ is the negative of the coefficient on $x_{t}$.

Assume, on the other hand, that one believes that seasonal fluctuations in the exogenous variables work their way through the system like all other fluctuations in the exogenous variables. In addition, suppose interest is focused on the ability of the seasonal fluctuations in the exogenous variables to explain the seasonality in the endogenous variables. Under these conditions, (25) would have to hold

$$
\underline{y}_{i}^{g}+\frac{H_{11}^{*} H_{12}}{\left|H_{11}\right|} \underline{x}_{t}^{g}=0
$$

This restriction arises from the fact that the true economic model exists between the observed series, and, therefore, the seasonal portion of $\underline{x}_{t}$ should explain the seasonal portion of $y_{t}$.

However, this approach still suffers from the problems of defining and obtaining an optimal adjustment and/or appropriate decomposition.

As indicated earlier, the approach taken in this paper is slightly different. The structural model is written in a manner which presumes that its form holds for the observed data and not only the trend component.

$$
H_{11} y_{t}+H_{12} \underline{x}_{t}=F_{11} \underline{e}_{1 t}
$$

The hypothesis to be tested is that seasonality enters the system through the process generating the exogenous variables. That is, the process generating $x_{t},(6)$, is written as a multiplicative seasonal time series model. By doing this, it is hoped to broaden the model by allowing a slightly greater flexibility with regard to the form of the seasonal fluctuation.

Since one of the objectives is to avoid choosing an arbitrary decomposition prior to developing an adequate model, a means must be devised by which conclusions can be drawn concerning the ability of the exogenous variables to account for the seasonality in the endogenous variables. Fortunately, there is a straightforward method of doing this. Since the process generating the $x_{t}$ 's will be associated, in general, with both seasonal AR and seasonal MA polynomials, it is possible to trace these polynomials through the analysis to determine their impact on the TF's and FE's of the system. Once the TF's and FE's have been obtained, they can be estimated and the results compared with the implications of the theory used in writing the structural model. To the extent that the estimated models are in agreement regarding the behavior of these seasonal polynomials, the hypothesis of exogenous seasonality will be accepted.

Proceeding in the manner previously described yields some interesting insights into the type of stochastic properties that are likely to be exhibited by the endoge. nous variables. Assume that (26) is written as a multivariate-multiplicative seasonal time series process.

$$
H_{22} S_{22} \underline{x}_{t}=F_{22} T_{22} \underline{e}_{2 t}
$$

where it is assumed that $S_{22}$ and $T_{22}$ are matrices having elements that are polynomials is $L^{s}$, where $s$ is the seasonal period. For simplicity, consider the case where the exogenous variables are independent so that $H_{22}, S_{22}$ $T_{22}$ and $T_{22}$ are all diagonal. This is sufficient to enable each and every exogenous variable to be written as a strictly multiplicative seasonal time series process.

Given (27) as the process generating $x_{l}$, a set of FE's can be obtained by substituting (27) in to (26) with the following result:

$$
\begin{gathered}
\left|H_{11}\right|\left|H_{22}\right|\left|S_{22}\right| y_{t}=-H_{11}^{*} H_{12} S_{22}^{*} H_{22}^{*} F_{22} T_{22} \underline{e}_{2 t} \\
+H_{11}^{*}\left|H_{22}\right|\left|S_{22}\right| \underline{e}_{1 t}
\end{gathered}
$$

Inspection of (28) reveals that the $A R$ portion of the processes for the endogenous variables will, in general, be in the form of the multiplicative seasonal model. However, the MA portion of (28) does not factor, in general, into the multiplicative form. Consequently, one might not, in general, expect to find the endogenous variables to be strictly multiplicative seasonal processes (i.e.. multiplicative in both the AR and MA portions). It would seem that the MA term would have characteristics of both multiplicative and additive seasonal variation. This implication will be investigated further in the economic model analyzed in the next section, and it will be seen that if certain restrictions are placed on the structure and on the processes generating the exogenous variables, (28) will become strictly multiplicative.

Although no mention has been made, up to this point. of constant terms or intercept terms, it is straightforward to see how they can be handled in the framework that has been discussed. If these intercept terms are considered constants, they can be carried along as deterministic elements of $\underline{x}_{t}$, or, if they are considered random and generated by a process, perhaps seasonal, they can again be considered as elements of $x_{t}$. In either case, the inclusion of these intercepts is a simple extension of the methodology outlined in this section.
To

follow

1. 1

2.

3.

4.

Mod

In

and

the

abol

riun

non

The

stn

fror

out

ger

\section{his}

the

(ol

or

\section{m.}

$\mathrm{Fi}$ 
To summarize, the approach that will be applied in the following sections is to-

1. Construct an economic model with an explicit specification of seasonality.

2. Derive the implied TF's and FE's of the model noting where the seasonal specification places restrictions on the form of these equations.

3. Empirically check these restrictions against the data.

4. Utilize the empirical results to suggest altemative specifications of the model if the model under consideration proves deficient.

\section{ANALYSIS OF AN ECONOMIC MODEL}

\section{Model Formulation}

In this section, a simple monetary model is formulated and analyzed to illustrate how the techniques outlined in the previous sections might be helpful in gaining insights about seasonality and its role in an economic model.

The economic model contains five variables: Equilibrium money stock, a measure of real income or wealth, nominal interest rate, price level, and the monetary base. The model is written to allow for various types of lag structures having form and length that are to be inferred from the data. In addition, no restrictions are placed on the theoretical elasticities and the growth rate of real output is allowed to vary. Expectations in this model are generated rationally in the sense of Muth [14]. That is, expectations are formed, based on information in the past history of the exogenous variables and the structure of the model. Finally, the monetary base and real income (output) are treated as exogenous or independently determined, and seasonality is assumed to enter the system only through these variables.

Obviously, in a simple model, such as this, there are many possible sources of specification error. However, this study focuses on two important aspects of the model. First, the assumption of the exogeneity of the monetary base and real income may not be an adequate representation. For instance, as specified, the model assumes that an open-loop control strategy has been adopted by the policy makers with regard to the creation of the monetary base. The alternative is, of course, some sort of closedloop control scheme, whereby the authorities respond to changes in the price level or interest rate in determining the growth of the base. The exogeneity of real income assumes the absence of a Phillips-curve relationship or feedback from the monetary sector to the real sector. ${ }^{10}$ Therefore, it is of interest to investigate the adequacy of the exogeneity assumptions in light of these other possible specifications of the model.

Secondly, seasonality is assumed to enter the model only through the exogenous variables. It may be that

${ }^{10}$ See Lucas [13] and Sargent [20] for a more thorough treatment of the issues surrounding this phenomenon. there are separate seasonal effects that enter directly through the money demand or money supply equations that are different from those induced by the seasonal influence of real income and the base. Such effects may be due to seasonally varying parameters in the structure. If this is the case, the empirical results would be at variance with the implications of the model.

The equations of the model include (1) a money demand equation, (2) a money supply equation, (3) a money market equilibrium condition, (4) the Fisher equation, and (5) a rational expectations equation. We can write these equations as follows:

$$
\begin{aligned}
M_{t}^{D} & =L\left(Y_{t}, i_{t}, P_{t}\right) \\
M S & =S\left(B_{t}\right) \\
M_{t} & =M_{t}^{D}=M S \\
i_{t} & =\rho_{t}^{*}+\pi_{t}^{*} \\
\pi_{t}^{*} & =E\left(\pi_{t} \cdot\right)
\end{aligned}
$$

where

$M_{t}^{D}=$ nominal money demand at time $t$

$M S_{t}^{S}=$ nominal money supply at time $t$

$Y_{t}=$ real income (output) at time $t$

$i_{t}=$ nominal interest rate at time $t$

$P_{t}=$ price level at time $t$

$B_{\imath}=$ net source base at time $t$

$\rho_{t}^{*}=$ anticipated real interest rate as of time $t$

$\pi_{i}^{*}=$ anticipated rate of inflation as of time $t$

Equation (33) builds the rational expectations hypothesis into the model, and $E\left(\pi_{t} \mid \cdot\right)$ denotes a conditional expectation of inflation given the equations of the model and past information."

It will be assumed that equation (29) can be written as

$$
r_{M_{t}}=\alpha_{t} r_{Y_{t}}+\beta_{t} \Delta i_{t}+\gamma_{1} r_{P_{t}}+u_{1 t}
$$

\footnotetext{
"Given the previous structure, there are many other issues that could also be raised. For example, most economists agree that permanent income, or possibly wealth, is a more appropriate income measure for the money demand function than real output. One might also consider an adjustment process rather than require market clearing at each time $t$. Finally, a more complicated money supply relationship might be considered to allow for changing reserve ratios, or changing interest rates that would affect the money multiplier. Clearly, a thorough examination of this model would have to consider these alternatives. However, the objective of this paper is somewhat less ambitious. Here, the intent is to gain a better understanding of the techniques and the issues surrounding seasonality.
} 
and that the money multiplier is nonautocorrelated so that (30) can be written as

$$
r_{M_{t}}=\alpha_{2} r_{B_{t}}+u_{2 t}
$$

where $\Delta=(1-L)$ is the difference operator; hence, $r_{k}=\Delta$ $\ell n(k)$ is the rate of growth of $k$. The coefficients are, in general, unrestricted in that they can be interpreted as polynomials in the lag operator. However, as a starting point, it will be assumed that they are constants, and the empirical results will be utilized to suggest alternative lag structures. ${ }^{12}$ For convenience, both $u_{1 t}$ and $u_{2 t}$ will be considered independent, nonautocorrelated disturbance terms.

The remainder of the model involves the Fisher equation and the rational expectations hypothesis. Since $\pi_{t}=\ln P_{t+1}-\ln P_{t}=\Delta \ln P_{t+1}=r_{P_{t+1}}$

$$
i_{t}=\rho_{t}^{*}+r_{P_{t+1}}^{*}
$$

and the expectation can be written as

$$
r_{P_{t+1}}^{*}=\underset{l}{E}\left(r_{P_{t+1}} \mid \cdot\right)
$$

At this point, some assumption must be made about $\rho_{t}^{*}$, the anticipated real rate of interest. In order to keep this analysis from becoming unduly complicated, the anticipated real rate will be considered a random variable with a constant expected value. Therefore,

$$
i_{t}=r_{P_{t+1}}^{*}+u_{3 t}
$$

where $u_{3 x}$ may have a nonzero mean. Of course, if the anticipated real rate were autocorrelated, then $u_{3 x}$ would also be autocorrelated. In addition, $u_{3 x}$ will be considered independent of $u_{1 t}$ and $u_{2 t}$.

Utilizing the assumption that the monetary base and real income are exogenous, the system can be completed by writing down the processes generating these variables.

$$
\begin{aligned}
& \phi_{B}(L) \Gamma_{B}\left(L^{12}\right) \Delta_{12} r_{B_{t}}=\theta_{B}(L) \Omega_{B}\left(L^{12}\right) u_{4} \\
& \phi_{Y}(L) \Gamma_{Y}\left(L^{12}\right) \Delta_{12} r_{Y_{t}}=\theta_{Y}(L) \Omega_{Y}\left(L^{12}\right) u_{5 t}
\end{aligned}
$$

where $\phi_{i}, \Gamma_{i}, \theta_{i}$, and $\Omega_{i}$ are polynomials in the lag operator having roots that satisfy the stationarity and invertibility conditions, and $\Gamma_{B}, \Gamma_{Y}, \Omega_{B}$ and $\Omega_{Y}$ represent the seasonal polynomials that are to be traced through the model.

Now that the model has been developed, the system represented by equations (34), (35), (37), and (38) can be

\footnotetext{
12 Whether differencing is appropriate for these structural relationships is not a real issue. The result of overdifferencing would be to induce moving average complications into the error structure that can be handled in the estimation procedure. (See Plosser and Schwert [18].)
}

rewritten in the form of a system of simultaneous equations as shown in (5), yielding ${ }^{13}$

$$
\begin{aligned}
{\left[\begin{array}{ccc}
1 & -v_{1} & -\beta_{1} \Delta \\
1 & 0 & 0 \\
0 & 0 & \Phi \Delta_{12}
\end{array}\right]\left[\begin{array}{c}
r_{M_{t}} \\
r_{P_{t}} \\
i_{t}
\end{array}\right] } \\
+\left[\begin{array}{cc}
0 & -\alpha_{1} \\
-\alpha_{2} & 0 \\
-\Psi_{1} \Delta_{12} & -\Psi_{2} \Delta_{12}
\end{array}\right]\left[\begin{array}{l}
r_{B_{t}} \\
r_{Y_{t}}
\end{array}\right]=\left[\begin{array}{l}
u_{1 t} \\
u_{2 t} \\
\Delta_{12} v_{t}
\end{array}\right]
\end{aligned}
$$

where

$$
\begin{aligned}
\Phi= & \left(\gamma_{1}-\beta_{1}\right) \\
\Psi_{1}= & \alpha_{2} \sum_{j=0}^{\infty}\left(\frac{-\beta_{1}}{\gamma_{1}-\beta_{1}}\right)^{j} \pi j+1 \\
\Psi_{2}= & -\alpha_{1} \sum_{j=0}^{\infty}\left(\frac{-\beta_{1}}{\gamma_{1}-\beta_{1}}\right)^{j} \pi_{j+1}^{n}(L) \\
\Delta_{12} \nu_{t}= & \Delta_{12} \sum_{j=0}^{\infty}\left(\frac{-\beta_{1}}{\gamma_{1}-\beta_{1}}\right)^{j} \\
& \left(E u_{2 t+j+1}-\beta_{1} E u_{3 t+j+1}-E u_{t} t+j+1\right.
\end{aligned}
$$

$u_{1 t}, u_{2 t}$, and $u_{3 t}$ are nonautocorrelated and independent disturbance terms. For convenience, let

$$
H_{11}=\left[\begin{array}{ccc}
1 & -\gamma_{1} & -\beta_{1} \Delta \\
1 & 0 & 0 \\
0 & 0 & \Phi \Delta_{12}
\end{array}\right]
$$

and

$$
H_{12}=\left[\begin{array}{cc}
0 & -\alpha_{1} \\
-\alpha_{2} & 0 \\
-\Psi_{1} \Delta_{12} & -\Psi_{2} \Delta_{12}
\end{array}\right]
$$

Through some simple algebraic manipulations, both the TF's and FE's can be written down, and the following analysis highlights some of the more interesting properties of the TF's and FE's. ${ }^{14}$

\section{Analysis of the Transfer Functions}

The TF's of the system are easily obtained by premultiplying both sides of (41) by $H_{11}^{*}$. Writing the resulting

${ }^{18}$ For the mathematical derivation of (41), the reader is referred to app. A.

14 See app. A for the derivations
PLOS.

systeı nous to

The base the

simp $\alpha_{2}$ is $\bmod$ estin the : only the I of 0 migl was the that mot ams wor pari bia! foll offs firs the to 1 ma

deg dist int wt po ce eq (A

$\Psi$ im th. in va C. m 
system of equations, one by one, the TF of each endogenous variable can be analyzed in greater detail.

The TF for the nominal money stock can be simplified to

$$
\Delta_{12} r_{M_{t}}=\alpha_{2} \Delta_{12} r_{B_{t}}+\Delta_{12} u_{2 t}
$$

The money supply is seen to be a function only of the base and real income does not enter as an input. Under the assumption that $\alpha_{2}$ is a constant coefficient, (45) is simply a regression model with moving average errors. If $\alpha_{2}$ is a polynomial in the lag operator, it is a distributed lag model. In either case, note that $\alpha_{2}$ can be directly estimated, using nonlinear techniques. In addition, if all of the seasonality in $M$ is explained by the base $(B)$, then the only evidence of seasonal autocorrelation should appear in the noise process as a seasonal moving average polynomial of order 1 and parameter value of 1 . Alternatively, there might be seasonal fluctuations in the money multiplier. As was noted earlier, the model has implicitly assumed that the multiplier is nonautocorrelated. However, to the extent that the Federal Reserve Board offsets changes in the money multiplier by either increasing or decreasing the amount of currency as it deemed appropriate, the result would be to force the first-order seasonal moving average parameter (SMA) away from 1 and to induce downward bias into the estimated value of $\alpha_{2}$. In fact, if the Fed. followed a policy of no money growth and only sought to offset the multiplier exactly, the estimate of $\alpha_{2}$ and of the first-order SMA parameter would be near zero.

The TF for prices is somewhat more complicated than the one describing money but, by that very fact, turns out to have interesting interpretations. Through some algebraic manipulations, the following expression is obtained:

$$
\begin{gathered}
\Delta_{12} r_{P_{t}}=\left(\frac{\alpha_{2} \Phi-\beta_{1} \Delta \Psi_{1}}{\gamma_{1} \Phi}\right) \Delta_{12} r_{B_{t}}+\left(\frac{-\alpha_{1} \Phi-\beta_{1} \Delta \Psi_{2}}{\gamma_{1} \Phi}\right) \Delta_{12} r_{Y_{t}} \\
+\Delta_{12}\left(-\Phi u_{1 t}+\Phi u_{2 t}-\beta_{1} \Delta v_{t}\right)
\end{gathered}
$$

The analysis of this expression will depend, to a large degree, on what can be said about the form of the distributed lag on $\Delta_{12} r_{B_{t}}$ and $\Delta_{12} r_{y_{t}}$. Fortunately, several interesting observations can be made. Consider the case where all the structural parameters in the model are polynomials of zero degree in $L$. Under these circumstances, the only polynomials in $L$ (other than the difference operators) arise from the terms $\Psi_{1}$ and $\Psi_{2}$. Note from equation (42), where $\Psi_{1}$ and $\Psi_{2}$ are defined, and equations $(A-19)$ and (A-20) in appendix $A$, that, in general, $\Psi_{1}$ and $\Psi_{2}$ will be polynomials which are infinite in length. The implication is that even though there are no lagged relationships specified in the structural model, that, due to the expectational aspect of the model, there exists an infinite distributed lag relationship between the exogenous variables and the endogenous variables of the system. Consequently, estimating this transfer function would, most likely, result in a rational distributed lag (RDL) model as a means of parsimoniously representing such a relationship.

Secondly, in appendix $A$ it is shown that the expressions for $\Psi_{1}$ and $\Psi_{2}$ involve the summation of varying powers and cross-products of the parameters in the seasonal and nonseasonal polynomials that are generating the exogenous variables. It is possible that the data would not indicate a need for seasonal parameters (i.e., specific coefficients at the seasonal lags) in the RDL formulation. If this is true, then the only evidence of seasonal autocorrelation appears in the error term as a seasonal moving average polynomial of order 1 with parameter value of 1 . The presence of $\Psi_{1}$ and $\Psi_{2}$ also indicate that, even though $\Delta_{12} r_{B_{t}}$ and $\Delta_{12} r_{y_{t}}$ may be seasonal, the existence of an expectations mechanism has a smoothing effect on the output variable $\Delta_{12} r_{p_{t}}$. This smoothing effect arises out of the infinite distributed lag relationship between the inputs and the output variable $\Delta_{12} r_{P_{t}}$. In other words, $\Delta_{12} r_{P_{t}}$ will be a weighted average of all past values of $\Delta_{12} r_{B_{1}}$ and $\Delta_{12} r_{Y_{1}}$.

An additional point of interest is how this model can simplify under alternative assumptions about the structural model. For example, if the classical quantity theory of money were true, then $\beta_{1}$ would equal zero, and $\gamma_{1}$ would equal 1 , allowing (46) to reduce to

$$
\Delta_{12} r_{P_{t}}=\alpha_{2} \Delta_{12} r_{B_{t}}-\alpha_{1} \Delta_{12} r_{Y_{t}}+\Delta_{12}\left[-u_{1 t}+u_{2 t}\right]
$$

In a similar manner, the TF for the nominal interest rate can be written as

$$
\Delta_{12} i_{t}=\left(\frac{\Psi_{1}}{\Phi}\right) \Delta_{12} r_{B_{t}}+\left(\frac{\Psi_{2}}{\Phi}\right) \Delta_{12} r_{Y_{t}}+\left(\frac{\Delta_{12}}{\Phi}\right) v_{t}
$$

Notice that here too, the distributed lags on $\Delta_{12} r_{B_{1}}$ and $\Delta_{12} r_{Y_{1}}$ will in general be infinite in length and, therefore, more easily modeled as a RDL even when the structural parameters only indicate contemporaneous relationships. As was pointed out, this is due to the expectations aspect of the model. In addition, if $\Psi_{1}$ and $\Psi_{2}$ do not display strong seasonal properties, the only evidence of seasonality that one would expect to find, if the model is correct, occurs in the error term of the form $\Delta_{12}$. Once again, it is worthy of note that because of $\Psi_{1}$ and $\Psi_{2}$ and the smoothing effect they have on $\Delta_{12} i_{t}$, the interest rate most likely would not display seasonal movements that are visually striking.

Table 1 summarizes the transfer functions for the model under consideration. Both a general formulation and a simplified formulation suggested by the classical quantity theory of money, as previously discussed, are presented for comparison.

\section{Analysis of the Final Equations}

The next set of equations to be analyzed are the final equations (FE's). They can be obtained, as indicated, in the subsection on the analysis of linear dynamic econo- 

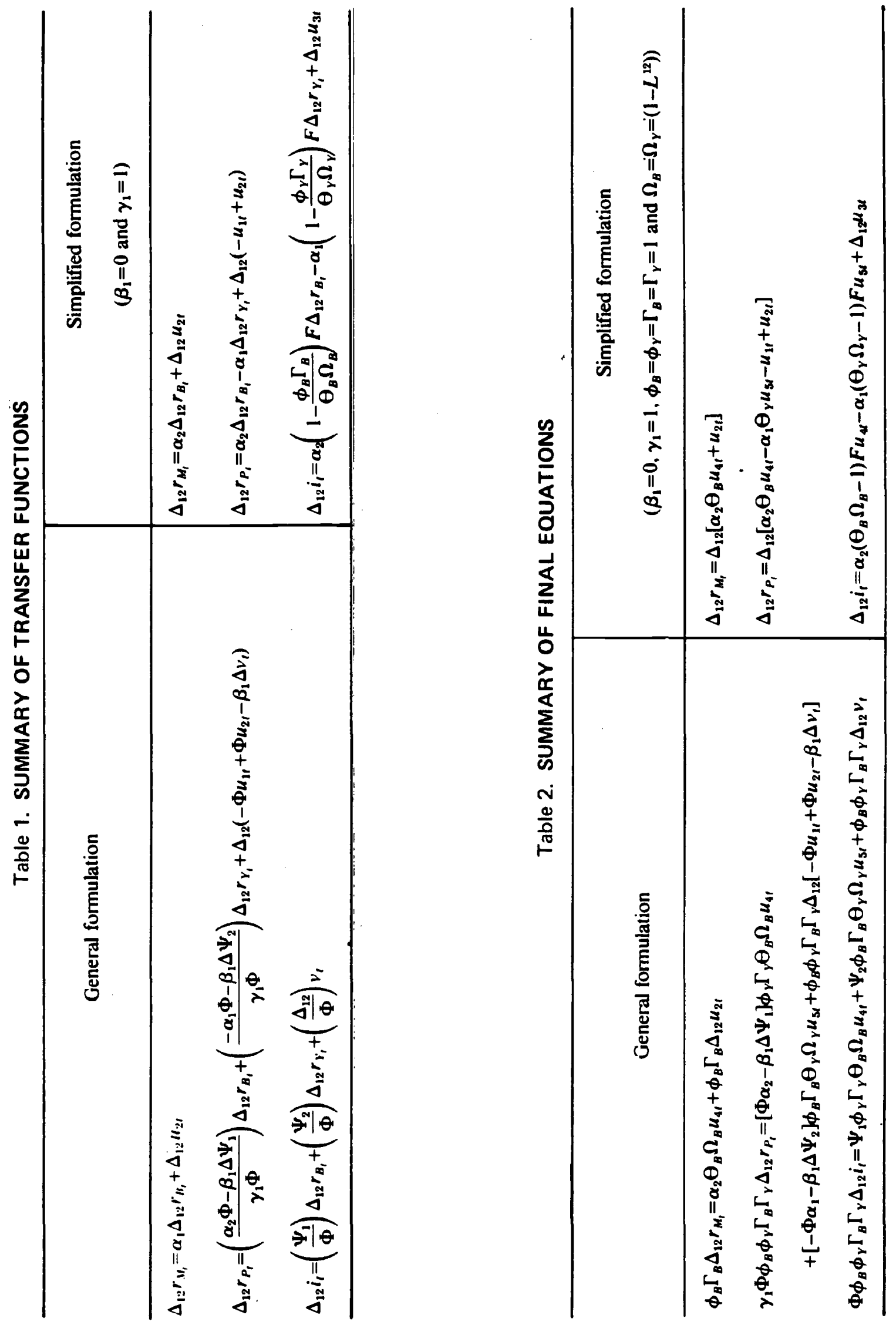
metric models. In deriving these equations, it is important to recognize that equations (39) and (40) are rewritten as

$$
\begin{gathered}
\Delta_{12}\left[\begin{array}{l}
r_{B_{t}} \\
r_{Y_{t}}
\end{array}\right]=\left[\begin{array}{cc}
\phi_{B} \Gamma_{B} & 0 \\
0 & \phi_{Y} \Gamma_{Y}
\end{array}\right]^{-1}\left[\begin{array}{cc}
\theta_{B} \Omega_{B} & 0 \\
0 & \theta_{Y} \Omega_{Y}
\end{array}\right] \cdot\left[\begin{array}{l}
u_{4 t} \\
u_{S t}
\end{array}\right] \\
=H_{22}^{-1} F_{22}\left[\begin{array}{l}
u_{4 t} \\
u_{S t}
\end{array}\right]
\end{gathered}
$$

This presumes the independence of $u_{4 t}$ and $u_{5 t}$, but such a restriction is not necessary. An alternative specification might allow the $(1,2)$ and $(2,1)$ elements of $F_{22}$ in $(49)$ to be nonzero. This would allow for a dynamic relationship among the inputs.

As derived in appendix A, the FE for the equilibrium money stock $(M)$ can be written as follows:

$$
\begin{gathered}
\gamma_{1} \Phi \phi_{B} \phi_{Y} \Gamma_{B} \Gamma_{Y} \Delta_{12} r_{M_{t}}=\gamma_{1} \Phi \alpha_{2} \phi_{Y} \Gamma_{Y} \theta_{B} \Omega_{B} u_{4} \\
+\phi_{B} \phi_{Y} \Gamma_{B} \Gamma_{Y} \nu_{1} \Phi \Delta_{18} u_{2 \ell}
\end{gathered}
$$

Notice that $\gamma_{1} \Phi \phi_{Y} \Gamma_{Y}$ can be factored out of both sides, leaving

$$
\phi_{B} \Gamma_{B} \Delta_{12} r_{M_{t}}=\alpha_{2} \Theta_{B} \Omega_{B} u_{4 t}+\phi_{B} \Gamma_{B} \Delta_{12} u_{2 t}
$$

The FE for the money stock is a function of the structural parameter $\alpha_{2}$, the error term $u_{2 y}$, and process generating the monetary base (B). More important is that, by introducting seasonality by way of the exogenous variables, seasonality is induced on the endogenous variable (M) and, in fact, on each and every endogenous variable in the system, as will be pointed out in subsequent analyses.

It is known (e.g., see [2]). that the sum of two moving average processes is representable as a single invertible linear process in one random variable. Consequently, given that $u_{2 t}$ and $u_{4 t}$ are independent due to the assumption that the monetary base is exogenous, the order of this moving average polynomial will be equivalent to the order of the expression $\alpha_{2} \Theta_{B} \Omega_{B}$ or $\phi_{B} \Gamma_{B} \Delta_{12}$, whichever is greater.

The FE for prices (P) is shown in appendix $A$ to be

$$
\begin{aligned}
\gamma_{2} \Phi \phi_{B} \phi_{Y} \Gamma_{B} \Gamma_{Y} \Delta_{12} r_{P_{t}}=\left[\Phi \alpha_{2}-\beta_{1} \Delta \Psi_{1}\right] \phi_{Y} \Gamma_{Y} \Theta_{B} \Omega_{B} u_{4 t} \\
+\left[-\Phi \alpha_{1}-\beta_{1} \Delta \Psi_{2}\right] \phi_{B} \Gamma_{B} \Theta_{Y} \Omega_{Y} u_{S t} \\
+\phi_{B} \phi_{Y} \Gamma_{B} \Gamma_{Y} \Delta_{12}\left[-\Phi u_{1 t}+\Phi u_{2 t}-\beta_{1} \Delta v_{t}\right]
\end{aligned}
$$

Once again, seasonality is seen to be induced on an endogenous variable only as a result of exogenous seasonality. This fact is evident from the presence of the $\Delta_{12}$ operator and the seasonal polynomials $\Gamma_{B}, \Gamma_{Y}, \Omega_{B}$, and $\Omega_{y}$. As occurred in the FE for money, the AR side of (52) is in the form of the multiplicative seasonal time series model, and the MA portion is not. In fact, the MA portion appears to border on the unintelligible. However, some insights can be obtained from this representation.
In order to gain some understanding of (52), suppose $\phi_{B}=\phi_{Y}=\Gamma_{B}=\Gamma_{Y}=1$ and that $\Omega_{B}=\Omega_{Y} \dot{=}\left(1-L^{12}\right)$, then (52) can be rewritten as:

$$
\begin{gathered}
\gamma_{1} \Phi \Delta_{12} r_{P_{t}}=\Delta_{12}\left[\left(\Phi \alpha_{2}-\beta_{1} \Delta \Psi_{1}\right) \Theta_{B} u_{4}\right. \\
\left.+\left(-\Phi \alpha_{1}-\beta_{1} \Delta \Psi_{2}\right) \Theta_{\gamma} u_{5 t}-\Phi u_{1 t}+\Phi u_{2 t}-\beta_{1} \Delta v_{t}\right]
\end{gathered}
$$

Equation (53) now appears to be in the terms of the general multiplicative time series model. However, it is not, because both $\Psi_{1}$ and $\Psi_{2}$ are expressions involving seasonal polynomials and are, in general, of infinite length. Therefore, it is convenient to consider two possible cases for this expression, when $\beta_{1}=0$ and $\beta_{1} \neq 0$.

Suppose that the classical quantity theory of money were to be considered. In that case, (53) reduces to

$$
\Delta_{12} r_{P_{t}}=\Delta_{22}\left[\alpha_{2} \Theta_{B} u_{4 t}-\alpha_{1} \Theta_{y} u_{5 t}-u_{1 t}+u_{2 t}\right]
$$

which is obtained by allowing the exogenous variables to have no AR polynomials and for the seasonality to approach the seasonal means problem as well as having $\beta_{1}=0$ and $\gamma_{1}=1$ (i.e., restricting the interest rate elasticity of the demand for money to zero and requiring demand for real cash balances to the homogeneous of degree zero in the price level). Notice that, once again, as the economic model is simplified, so is the implied stochastic structure of the output variables of the system.

The implication of (54) is that the seasonally differenced rate of inflation would be a pure MA process. It would be in the form of the multiplicative seasonal model with the seasonal moving average polynomial of order 1 and parameter value close to 1 .

As was noted previously, the model has been carried through under the assumption that the $u$ 's in (54) are independent of one another. Under such an assumption the order of the monthly MA process would be of the order of $\alpha_{2} \Theta_{B}$ or $\Theta_{Y}$, whichever is larger. However, $u_{4 t}$ and $u_{5 t}$ may not be independent either contemporaneously or through time and similarly for $u_{1 t}$ and $u_{2 t}$.

Neither of these complications would alter the basic economics of the model but could affect the orders of the MA portions of the FE's. Therefore, if the classical quantity theory of money is true, one might expect to observe an ARIMA model for the natural log of prices to be of the form $(0,1, q)(0,1,1)_{12}$, where $q$ is determined by $\theta_{Y}, \theta_{B}$ and the covariance structure between the error terms.

The second case of (53) to be considered allows $\beta_{1}$ to be different from zero. In order to gain insight into this case, it is necessary to analyze the expressions for $\Psi_{1}$ and $\Psi_{2}$ in greater detail. Rewriting (53) yields

$$
\begin{aligned}
& \gamma_{1} \Phi \Delta_{12} r_{P_{t}}=\Delta_{12}\left[\Phi \alpha_{2} \Theta_{B} u_{4}-\Phi \alpha_{1} \Theta_{Y} u_{s t}-\Phi u_{1 t}\right. \\
& \left.+\Phi u_{2 t}-\beta_{1} \Delta v_{t}\right]-\Delta_{12} \beta_{1} \Delta\left[\Psi_{1} \Theta_{B} u_{4 t}+\Psi_{2} \Theta_{Y} u_{s t}\right]
\end{aligned}
$$


or

$$
\begin{gathered}
\gamma_{1} \Phi \Delta_{12} r_{P_{t}}=W_{t}-\Delta_{12} \beta_{1} \Delta \sum_{j=0}^{\infty}\left(\frac{-\beta_{1}}{\gamma_{1}-\beta_{1}}\right)^{j}\left[\alpha_{2} \pi_{j+1}^{(B)}(L) \Theta_{B} u_{4 t}\right. \\
\left.-\alpha_{1} \pi_{j+1}^{(Y)}(L) \Theta_{Y} u_{3 t}\right]
\end{gathered}
$$

Now, under the assumption that the structural coefficients are just constants, $W_{l}$ is a finite MA polynomial of order equal to the maximum order of $\Delta, \theta_{B}$ or $\theta_{Y}$, with seasonal polynomial $\Delta_{12}$. The second term is more complicated.

The expressions $\pi_{j+1}^{(B)}(L)$ and $\pi_{j+1}^{(Y)}(L)$ merely represent the weighting scheme applied to the infinite past history of $\Delta_{12} r_{B_{t}}$ and $\Delta_{12} r_{Y_{t}}$, respectively, to obtain the forecast of these variables at time $t$, for time $t+j+1$. This would imply that the FE for prices would involve an infinite MA polynomial. It is very difficult to evaluate the form of this polynomial for anything except the most trivial cases. However, if either $\theta_{B}, \theta_{Y}, \Omega_{B}$, or $\Omega_{Y}$ are of degree greater than zero, then the polynomial will be of infinite length. In finite samples, this infinite MA model may be indistinguishable from a more parsimonious AR representation. If the decay of this infinite MA is very slow, then one might even be led into differencing the series or estimating an AR polynomial that had a root close to the unit circle. It is even more interesting to note that the presence of $\Psi_{1}$ and $\Psi_{2}$ is due to the necessity of generating expectations and has an apparent smoothing effect on the autocorrelation structure of $\Delta_{12} r_{P_{t}}$, resulting in the seasonality in prices that appears much less pronounced.

The last FE to be considered is the one implied for the nominal interest rate $(i)$. It can be written as follows:

$$
\begin{aligned}
& \gamma_{1} \Phi \phi_{B} \phi_{Y} \Gamma_{B} \Gamma_{Y} \Delta_{12} i_{t}=\gamma_{1} \Psi_{1} \phi_{Y} \Gamma_{Y} \theta_{B} \Omega_{B} u_{4 t} \\
& +\gamma_{1} \Psi_{2} \phi_{B} \Gamma_{B} \theta_{Y} \Omega_{Y} u_{5 t}+\phi_{B} \phi_{Y} \Gamma_{B} \Gamma_{Y} \gamma_{1} \Delta_{12} v_{t}
\end{aligned}
$$

As has occurred for money and prices, seasonality has occurred in the nominal interest rate. In addition, the right-hand side of (57) does not indicate that a multiplicative time series model is the correct representation of the data if the model is true but that some mixture of the multiplicative and additive models would be more appropriate. However, if it is assumed that $\Gamma_{B}=\Gamma_{Y}=1$ and $\Omega_{B} \dot{=}$ $\Omega_{Y}=\Delta_{12}$, then (57) can be rewritten as

$$
\Phi \phi_{B} \phi_{Y} \Delta_{12} i_{t}=\Delta_{12}\left[\Psi_{1} \phi_{Y} \Theta_{B} \mu_{4 t}+\Psi_{2} \phi_{B} \Theta_{Y} u_{5 t}+\phi_{B} \phi_{Y} v_{t}\right]
$$

or allowing $\phi_{B}=\phi_{Y}=1$, as

$$
\Phi \Delta_{12} i_{t}=\Delta_{12}\left[\Psi_{1} \theta_{B} u_{4 t}+\Psi_{2} \theta_{Y} u_{5 t}+v_{t}\right]
$$

Notice that the terms $\Psi_{1}$ and $\Psi_{2}$ appear here as they did in the FE for prices. Consequently, if $\beta_{1} \neq 0$, then the data may indicate the need for an AR polynomial for $\Delta_{12} i_{\imath}$. In addition, if $\Psi_{1}$ and $\Psi_{2}$ imply weights that decline very slowly, then $\Delta_{12} i_{t}$ may appear nonstationary in finite samples. Similarly, the presence of $\Psi_{1}$ and $\Psi_{2}$, most likely, indicates that the seasonality in the interest rate is greatly attenuated.
Alternatively, simplifications of the economic model naturally leads to a simplification of the stochastic structure of $\Delta_{12} i_{t}$. If $\beta_{1}=0$, i.e., the classical quantity theory is true, with some algebraic manipulation, (59) reduces to

$$
\Delta_{12} i_{t}=\alpha_{2}\left(\theta_{B} \Omega_{B}-1\right) F u_{4 t}-\alpha_{1}\left(\theta_{Y} \Omega_{Y}-1\right) F u_{5 t}+\Delta_{12} u_{3 t}
$$

where $u_{3 t}$ is obtained from the expression for $\Delta_{12} v_{t}$ in (42) and $F$ is the forward shift operator so that $F^{j} z_{t}=z_{t+j}$. Therefore, the univariate model for the nominal interest rate might well be expected to follow something similar to a $(1,0, q)(0,1,1)_{12}$ or $\left(0,1, q^{\prime}\right)(0,1,1)_{12}$ process, where $q$ and $q^{\prime}$ would be determined by $\theta_{B}$ and $\theta_{Y}$ and the covariance between $u_{4 l}$ and $u_{5 t}$.

A summary of the FE's discussed in this section are presented in table 2. For comparison, both the general and the simplified versions are presented.

FE's and the census $X-11$ adjustment procedure-In light of the work done by Cleveland [5], who found a stochastic model for which the $\mathrm{X}-11$ procedure is nearly optimal in the sense of conditional expectation, it is interesting to analyze the stochastic structure implied by the economic model to see if and when the model might imply a structure for which the X-11 method, for example, is appropriate. The model developed by Cleveland is

$$
\begin{gathered}
(1-L)\left(1-L^{12}\right) y_{t}=\left(1-0.28 L+0.27 L^{2}+0.24\left(L^{3}+\ldots+L^{8}\right)\right. \\
+0.23 L^{9}+0.22 L^{10}+0.16 L^{11}-0.50 L^{12} \\
\left.+0.34 L^{13}+0.07 L^{14}\right) c_{t}
\end{gathered}
$$

where $c_{t}$ is a white-noise error term. ${ }^{15}$ This suggests that for data having an autoregressive structure $(1-L)\left(1-L^{12}\right)$ and having a moving average structure of length 14 and similar to that specified in (61), the $X-11$ procedure may do a fairly accurate job of decomposition.

Consider, for example, the FE for the money stock. From (61) and (51), it can be seen that, if the economic model is correct and if $\phi_{B}$ and $\Gamma_{B}$ are identically equal to 1 , then

$$
\Delta_{12} r_{M_{t}}=\alpha_{2} \Theta_{B} \Omega_{B} u_{4 t}+\Delta_{12} u_{2 t}
$$

or

$$
(1-L)\left(1-L^{12}\right)-n M_{t}=T(L) u_{t}
$$

where $T$ is at least of order 12 and maybe higher depending on the order of $\alpha_{2} \Theta_{B} \Omega_{B}$. Equation (63) suggests that the $\mathrm{X}-11$ procedure may provide a satisfactory decomposition of $\ell n M_{t}$ under some restrictions on the behavior of the exogenous variables. Though $T(L)$ is not likely to conform exactly to the MA process described in (61), the AR position is identical. On the other hand, if $\phi_{B}$

\footnotetext{
15 That is, for stochastic processes very similar to the one he derives, the seasonally adjusted data created by the $X-11$ can be considered approximately equal to the conditional expectation of a trend component, given the observed series.
}

PLO'

or $\Gamma$

auto

duct

$\mathrm{T}$

and

pric

lag

AR

pro

app

F

An

all

sea

on

por

sor

$\mathrm{mi}$

$\mathrm{mL}$

Th

mi

ho

thi

FI

to

(5 
or $\Gamma_{B}$ are not one, i.e., if the exogenous variables display autoregressive properties, the $\mathrm{X}-11$ procedure could produce grossly inaccurate results.

This analysis can also be done with the FE for prices and the interest rate. Consider equation (53) as the FE for prices. If $\gamma_{1} \Phi=\gamma_{1}\left(\gamma_{1}-\beta_{1}\right)$ is not a constant (i.e., contains a lag structure), the economic model would be indicating AR polynomials and, hence, a departure from the type of process for which the $\mathrm{X}-11$ procedure is considered appropriate.

FE's and Box-Jenkins multiplicative seasonal modelsAn additional point of interest is that the AR portion of all the FE's are already in the form of the multiplicative seasonal time series model, discussed in the subsection on seasonality in time series data. However, the MA portions do not appear to factor into seasonal and nonseasonal polynomials. In fact, the models, in general, imply a mixture type of model that contains some aspects of a multiplicative nature and others of an additive nature. This suggests that the properties of this type of mixed model should be investigated as a starting point for developing methods of adjustment. It would be of interest, however, to determine a set of conditions under which the theory would predict the multiplicative model. For the FE for the money stock, a sufficient set of conditions is to let $\Omega_{B} \doteq \Delta_{12}=\left(1-L^{12}\right)$ and $\Gamma_{B}=1$, which yields, from (51),

$$
\phi_{B} \Delta_{12} r_{M_{t}}=\Omega_{B}\left(\alpha_{2} \Theta_{B} u_{4 t}+\phi_{B} u_{2 t}\right)
$$

Finally, if $\phi_{B}=1,(64)$ reduces to a very simple pure seasonal moving average model

$$
\Delta_{12} r_{M_{t}}=\Omega_{B}\left(\alpha_{2} \Theta_{B} u_{4 t}+u_{2 t}\right)
$$

These assumptions are equivalent to stating that the process generating the monetary base has no autoregressive polynomials associated with it, neither seasonal nor nonseasonal, and that the seasonality in the base is very close to following the seasonal means model. (See the subsection on seasonality in time series data.) Recalling that $u_{4 t}$ and $u_{2 t}$ are assumed independent, and, considering the case where $\alpha_{2}$ is just a constant, the right-hand side of (56) reduces to a monthly MA polynomial having a degree that is equal to the degree of $\Theta_{B}$ and a seasonal polynomial of first-degree and parameter value of approximately 1 . Under such circumstances, the model implied for the natural $\log$ of money would be written as $(0,1, q)(0,1,1)_{12}$, where $q$ depends on the properties of $\Theta_{B}$.

Similarly, equation (54) represents a multiplicative formulation for the $\mathrm{FE}$ for the price variable. In this case, both $\Omega_{B}$ and $\Omega_{Y}$ need to approximately equal $\left(1-L^{12}\right), \Gamma_{B}$, and $\Gamma_{Y}$ equal to 1 , and, in addition, the quantity theory of money must hold so that $\beta_{1}=0$ and $\gamma_{1}=1$.

FE's and dependence of seasonality on structural assumptions-Because the FE for the money stock is reasonably simple, it is instructive to investigate it further. In particular, consider the effects on key aspects of the autocorrelation structure of $\Delta_{12} r_{M_{t}}$ under some different assumptions about the polynomials and parameters on the right-hand side of (62).

$$
\Delta_{12} r_{M_{t}} \equiv w_{t}=\alpha_{2} \Theta_{B} \Omega_{B} u_{4 t}+\Delta_{12} u_{2 t}
$$

Assume that the base is truly exogenous, i.e., the model is correct so that

$$
E\left(u_{4 t} u_{2 t-k}\right)=0 \text { for all } k
$$

By assumption,

$$
E\left(u_{4 t} u_{4 t-k}\right)=\left\{\begin{array}{l}
\sigma_{4}^{2} \text { if } k=0 \\
0 \text { if } k \neq 0
\end{array}\right.
$$

Although it has been assumed, so far, that $u_{2 t}$ is serially uncorrelated, it is interesting to relax this assumption somewhat. Recall that in this model $u_{2 t}$ incorporates changes in the money multiplier. Now, the money multiplier may have seasonal properties that are unspecified here. In order to keep the problem manageable, assume that changes in the money multiplier are random except for a seasonal effect. That is, assume that

$$
E\left(u_{2 t} u_{2 t-k}\right)=\left\{\begin{aligned}
\sigma_{2}^{2} & \text { if } k=0 \\
\gamma_{12}^{(2)} & \text { if } k=12 \\
0 & \text { otherwise }
\end{aligned}\right.
$$

which implies that changes in the multiplier follow a seasonal MA(1) process. If the multiplier were nonautocorrelated, then, of course, $\gamma_{12}^{(2)}=0$. Finally, assume that

$$
\Theta_{B}=(1-\theta L)
$$

and

$$
\Omega_{B}=\left(1-\Omega L^{12}\right)
$$

Under these assumptions, the variance of $\Delta_{12} r_{M_{t}}$ or $w_{t}$ can be shown to be

$$
\gamma_{0}^{(w)}=\alpha_{2}^{2}\left(1+\Theta^{2}\right)\left(1+\Omega^{2}\right) \sigma_{4}^{2}+2\left(\sigma_{2}^{2}-\gamma_{12}^{(2)}\right)
$$

A convenient method of getting an idea of how different assumptions affect seasonality is to investigate the autocorrelation coefficient of $w_{t}$ at lag 12. The autocovariance of $w_{t}$ at lag 12 is simply

$$
\gamma_{12}^{(w)}=-\alpha_{2}^{2} \Omega\left(1+\Theta^{2}\right) \sigma_{4}^{2}-\sigma_{2}^{2}+2 \gamma_{12}^{(2)}
$$

and the autocorrelation coefficient

$$
\begin{aligned}
\rho_{12}^{(w)}=\frac{\gamma_{12}^{(w)}}{\gamma_{0}^{(w)}} & =\frac{-\alpha_{2}^{2} \Omega\left(1+\Theta^{2}\right) \sigma_{4}^{2}-\sigma_{2}^{2}+2 \gamma_{12}^{(2)}}{\alpha_{2}^{2}\left(1+\Theta^{2}\right)\left(1+\Omega^{2}\right) \sigma_{4}^{2}+2\left(\sigma_{2}^{2}-\gamma_{12}^{(2)}\right)} \\
& =\frac{\left.-\alpha_{2}^{2} \Omega\left(1+\Theta^{2}\right) h-1+2 \rho_{12}^{(2)}\right)}{\alpha_{2}^{2}\left(1+\Theta^{2}\right)\left(1+\Omega^{2}\right) h+2\left(1-\rho_{12}^{(2)}\right)}
\end{aligned}
$$

where $h=\sigma_{4}^{2} / \sigma_{2}^{2}$ and $\rho_{12}^{(2)}=\gamma_{12}^{(2)} / \sigma_{2}^{2}$. 
If $\rho_{12}^{(2)}=0$ and $\Omega=1$, then it is clear that $\rho_{12}^{(w)}$ is known with certainty, since the process for the money stock is simply the dummy variable case. That is,

$$
\rho_{12}^{(\mu)}=-\frac{1}{2} \cdot \frac{\alpha_{2}^{2}\left(1+\theta^{2}\right) h+1}{\alpha_{2}^{2}\left(1+\theta^{2}\right) h+1}=-\frac{1}{2}
$$

However, if there is seasonality in the multiplier, meaning $\rho_{12}^{(2)} \neq 0$, then the implied value of $\rho_{12}^{(w)}$ is

$$
\rho_{12}^{(w)}=-\frac{1}{2} \cdot \frac{\alpha_{2}^{2}\left(1+\Theta^{2}\right) h+1-2 \rho_{12}^{(2)}}{\alpha_{2}^{2}\left(1+\theta^{2}\right) h+1-\rho_{12}^{(2)}}
$$

which, for $\rho_{12}^{(2)}>0$, is greater than $-\frac{1}{2}\left(\right.$ or $\left.\left|\rho_{12}^{(2)}\right|<\frac{1}{2}\right)$, even though $\Omega=1$.

Assume that the Fed. was interested in creating the simplest seasonal pattern possible in the money supply. If they knew the parameter $\alpha_{2}$ and the stochastic structure of the money multiplier ( $\sigma_{2}^{2}$ and $\gamma_{12}^{2}$ in this case), then values of $\theta, \Omega$, and $\sigma_{4}^{2}$ could be chosen to obtain a $\rho_{12}^{(w)}$ of $-\frac{1}{2}$, which would imply that the seasonal pattern in the money supply was merely a stable seasonal mean. It would then be straightforward to either adjust the money supply or, for the Fed., to design an optimal control scheme to effectively eliminate seasonality in the money supply.

\section{Summary}

In this section, the basic framework of a simple monetary model was postulated. Explicit assumptions were made regarding several important aspects of the model. First, the assumptions were made that the monetary base and real income are exogenous inputs to the system. This places theoretical restrictions on the covariance matrix between these variables and the endogenous variables of the system that can be checked against the data. Another issue of importance is the question of whether the economic structure generates seasonal fluctuations or only acts as a transmitter of seasonality. In order to shed light on this issue, it was hypothesized that seasonality enters the system only through the exogenous variables. This approach would be consistent with the system only transmitting seasonality. It was shown that this resulted in seasonality being induced into each and every endogenous variable and the FE's and, more importantly, the TF's obtained from the model display restrictions concerning the location and magnitude of certain seasonal parameters and polynomials. An important point to make concerning the FE is that, due to cancellation, the AR portion of the endogenous variables is not identical. Therefore, the estimated univariate models should not be restricted to have the same AR polynomials in the empirical work.

In addition, the theory suggests that, in general, the multiplicative seasonal model is not implied by the struc- ture. Instead, a more general structure is suggested that contains both additive and multiplicative characteristics. The model was then investigated in order to ascertain a set of assumptions sufficient to allow the theory to predict that a multiplicative seasonal model would be adequate in describing the FE's. It was found that, as the seasonality in the exogenous variables approached the simple seasonal means case and as the economic structure approached the classical quantity theory of money, the FE's approach a special case of the multiplicative seasonal model, or the seasonal means case. These results indicate that decomposition schemes, based on the general multiplicative time series model, would be inappropriate, since they are not suggested by the economic structure. In fact, it is clear that the multiplicative seasonal model will not, in general, result from linear models.

Another point investigated in this section was when the economic model implied that the stochastic behavior of the output variables would be of a form, similar to that suggested by Cleveland, which might be appropriate for decomposition by the $\cdot \mathrm{X}-11$ procedure. Finally, it was shown how an economic model can explain explicitly why seasonality in interest rates and prices does not appear to be important. The existence of an expectations mechanism has an attenuating effect on the seasonality and the autocorrelation structure of these series.

\section{EMPIRICAL RESULTS}

The purpose of this section is to demonstrate how one might utilize available data to test a theoretical economic model, such as the one outlined and analyzed in the previous section.

\section{Analysis of the Univariate Time Series}

In this section, the results from the analysis of the univariate time series properties of the raw or unadjusted data for each variable in the model are reported and compared with the implications of the FE's, as discussed in previous sections. The techniques used are essentially those developed by Box and Jenkins for the analysis of time series data as well as several other techniques, including likelihood ratio tests and posterior odds ratios, as utilized by Zellner and Palm [26; 27] and Zellner [25]. In general, interest centers on identifying and estimating models in the form, described in the subsection on seasonality in time series data,

$$
\phi_{p}(L) \Gamma_{p}\left(L^{s}\right) \Delta_{s}^{D} \Delta^{d} z_{t}=\theta_{q}(L) \Omega_{q}\left(L^{s}\right) a_{t}
$$

written as an ARIMA model of order $(p, d, q)(P, D, Q)_{s}$. It is assumed that $a_{t}$ is white noise and that the roots of $\phi(\xi)=0$ and $\theta(\xi)=0$, the monthly polynomials and $\Gamma(\xi)=0$ and $\Omega(\xi)=0$, the seasonal polynomials lie outside the unity circle so that $w_{t}=\Delta^{d} \Delta_{t}^{D} z_{t}$ is stationary and invertible.

It is important to note that, for a stationary series, the autocorrelations approach zero as the lag increases, so tions a encing is that for a I series remair stable would

Hor priates not re that $\mathrm{n}$ vecto statist tional wouls dures is to in thi of 1 , indic must that. stani asyn sizes distr the eter wor ider tims

finit que on aris inte.

con

all

of

the

\section{sal} thi
thi$$
\text { a }
$$ 
that persistently high values for the estimated autocorrelations at increasing lags might suggest the need for differencing. In addition, and a point that is often overlooked, is that sample autocorrelations need not have large values for a nonstationary series. All that is required is that the series generate a sample autocorrelation function that remains relatively flat. Similarly, a persistence of high or stable values at lags $12,24, \ldots$, etc. (with monthly data) would suggest the need for annual or seasonal differencing.

However, in many instances, the question of the appropriateness of differencing or the question of stationarity is not readily resolved. Unfortunately, tests and test statistics that rely on the asymptotic distribution of the observation vector are questionable, since the distribution of these statistics, when the series exhibits homogeneous nonstationarity, is generally not known. ${ }^{16}$ In light of this, it would seem inappropriate to use standard testing procedures to test for stationarity. One alternative to consider is to proceed with differencing and test for a root of one in the resulting model's MA polynomial. If there is a root of 1 , the process becomes noninvertible, and there is an indication of overdifferencing. ${ }^{17}$ However, two caveats must be mentioned here. First, Nelson [15] has shown that, for the first-order moving average process, the standard error of the parameter estimate, based on an asymptotic normal distribution, is understated in sample sizes as large as 100 . In addition, it is not clear what the distributional properties of the standard tests are under the null hypothesis, i.e., when the moving average parameter equals 1. Consequently, the approach followed in this work has been to utilize the standard techniques for the identification of the ARIMA models, while, at the same time, being aware of the problems that might arise in finite samples when the stationarity of the series is in question. Recall that, based on the theory in the section on the analysis of an economic model, this problem may arise with both the model for prices and the model for interest rates.

It is useful, ar his point, to make a few comments concerning the data being used in this analysis. As with all other econometric work, there is the recurring problem of finding data that adequately measure the quantities which are of theoretical interest. In this case, even the theoretical quantities are, in some instances, not universally agreed upon, such as the appropriate definition of the money stock, the appropriate measure of income, and the use of short vs. long-run interest rates.

The actual data used in this study are detailed in appendix $B$. The series are made up of monthly observations from January 1953 through July 1971. The net source base, as calculated by the St. Louis Federal Reserve Bank, is used as the unadjusted monetary base. The money stock is represented by M1, currency plus demand deposits. The interest rate is the yield on 1month Treasury bills, as compiled by Fama [7]. These

\footnotetext{
${ }^{18}$ See White [24] and Anderson [1].

${ }^{17}$ See Plosser and Schwert [18].
}

data should constitute reliable measures of the theoretical quantities. The remaining two series are somewhat less reliable measures for the variables of interest. The price level is represented by the Consumer Price Index (CPI) and real income (output) is measured by the Index of Industrial Production (IIP). Both of these measures are apt to contain measurement error by the mere fact that they are indexes. Sampling properties of these indexes might also cause problems, because the individual components of each index are not measured every month.

Plots of the raw data are presented in figures 3-7. Upon inspection of these charts, it becomes apparent why the issue of the appropriate level of differencing becomes difficult. In particular, the growth rates of the monetary base, M1, and the CPI seem to be increasing steadily throughout the time period. However, this does not appear as strikingly in the IIP. The interest rate appears nonstationary or highly autoregressive, which was noted in the theoretical discussions as something that might be observed.

Table 3 summarizes the results of a univariate time series analysis of the different series. ${ }^{18}$ The first two series, $\ln (B)$ and $\ln (Y)$, represent the exogenous variables in the system, and the models shown in table 3 describe the processes governing them. These findings indicate that there are no autoregressive polynomials associated with the exogenous variables and that the moving average polynomials are of low order. In terms of the notation of the model, $\phi_{B}=\Gamma_{B}=\phi_{Y}=\Gamma_{Y}=\theta_{B}=1$, $\Omega_{B}=\left(1-0.897 L^{12}\right), \quad \Omega_{Y}=\left(1-0.915 L^{12}\right), \quad$ and $\theta_{Y}=\left(1+0.247 L+0.157 L^{2}\right)$.

An analysis of the $\ln (M)$ reveals that an $(0,1,3)$ $(0,1,1)_{12}$ appears as an adequate representation of the data. (Note that this is the same model used in generating the forecasts in the first section). Since this variable is an endogenous variable in the system, the next step is to interpret these results in light of the theoretical FE's implied by the model, equation (51). Since the SMA1 parameter for this model is much less than 1, it appears that if the theory is correct, the model can not be factored exactly, so the multiplicative model is at best an approximation. Also, since $u_{4 t}$ in (51) is nonseasonal by construct, the fact that the seasonality is slightly different in the process for $r_{M_{t}}$ and $r_{B_{t}}$ indicates the possibility of seasonal influences from $u_{2 t}$. As noted and discussed earlier, this could be due to seasonal fluctuations in the multiplier.

Another point is that the monthly MA polynomial is of order 3 in this case, and, under the assumption of constant coefficients in the structure, the theory suggested that the order should be the same as the order of $\theta_{B}$, which is zero. This might suggest that something else is

\footnotetext{
18 These calculations, as well as many others in this paper. were performed using a set of time-series programs developed by $C$. $R$. Nelson, S. Beveridge, and G. W. Schwert, Graduate School of Business, University of Chicago. The reader is referred to Plosser [17] for a more complete documentation of the development of these results.
} 


\begin{tabular}{|c|c|c|c|c|c|c|c|}
\hline & 表 & a & 8 & 8 & $\stackrel{0}{0}$ & 8 & 8 \\
\hline & 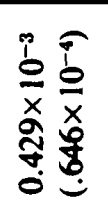 & 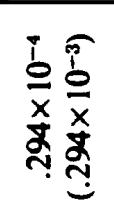 & 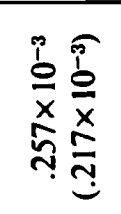 & 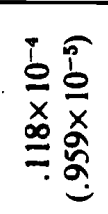 & 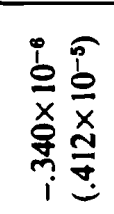 & 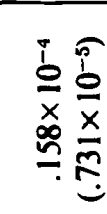 & 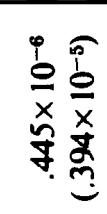 \\
\hline$\sum_{n=1}^{\bar{x}}$ & వీ & $\frac{n}{a} \bar{a}$ & बे. & 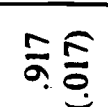 & $\bar{s} \bar{g}$ & 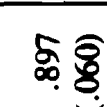 & 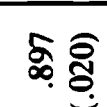 \\
\hline
\end{tabular}

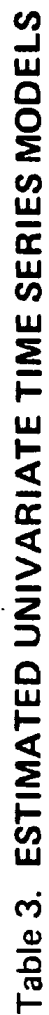

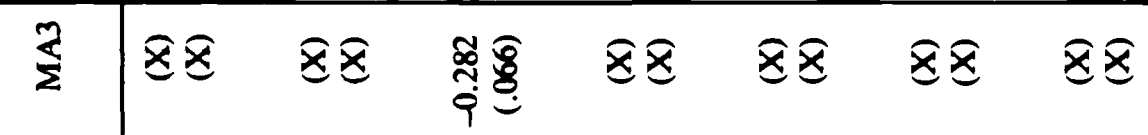

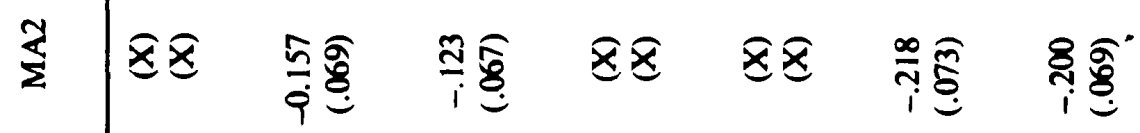

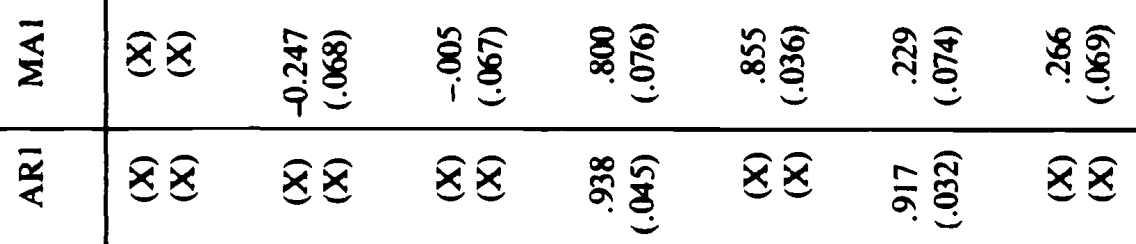

\begin{tabular}{|c|c|c|c|c|c|c|}
\hline$\tilde{r}_{b}^{*}$ & $\begin{array}{l}\frac{i}{\bar{o}} \\
\underline{x} \\
\underline{\underline{N}}\end{array}$ & $\begin{array}{l}0 \\
\frac{b}{x} \\
\hat{n} \\
=\end{array}$ & $\begin{array}{l}\frac{j}{x} \\
\frac{\delta}{g} \\
g\end{array}$ & 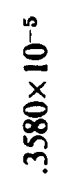 & 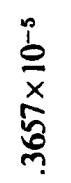 & $\begin{array}{l}\tilde{\delta} \\
\bar{x} \\
b \\
0 \\
0\end{array}$ \\
\hline
\end{tabular}

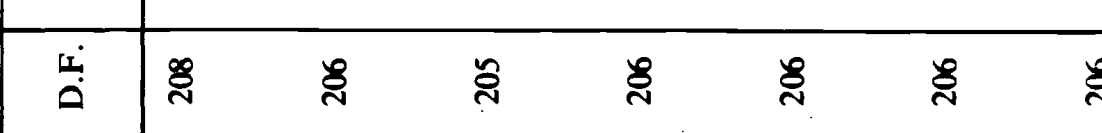

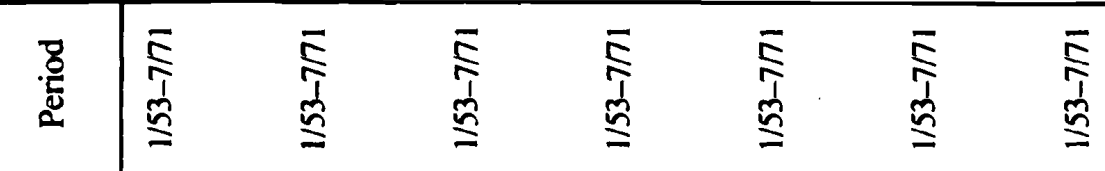

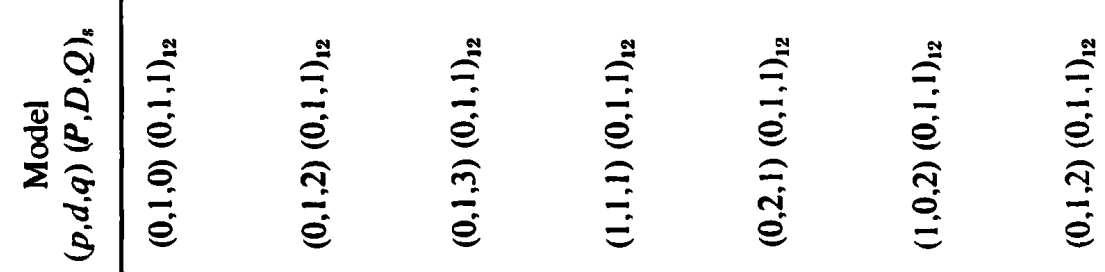

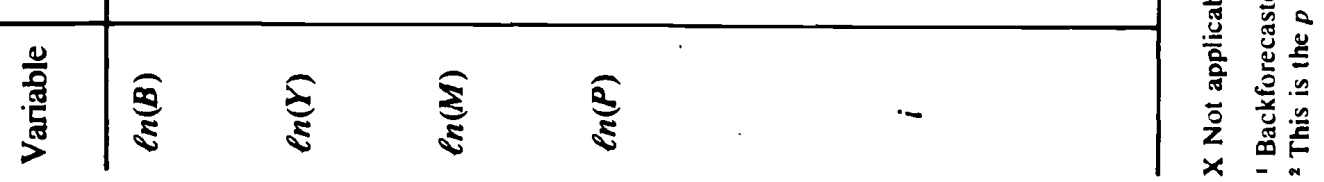




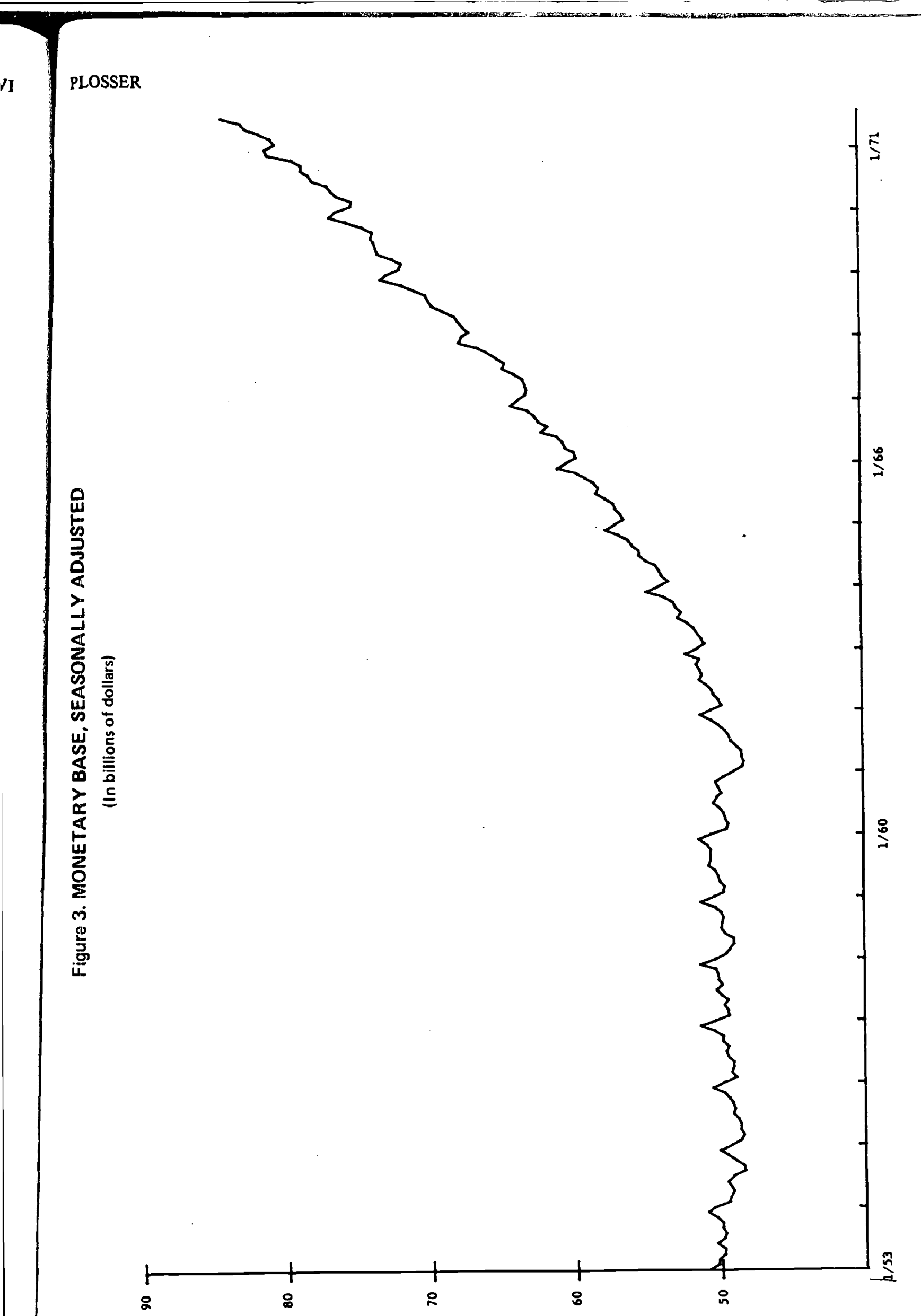




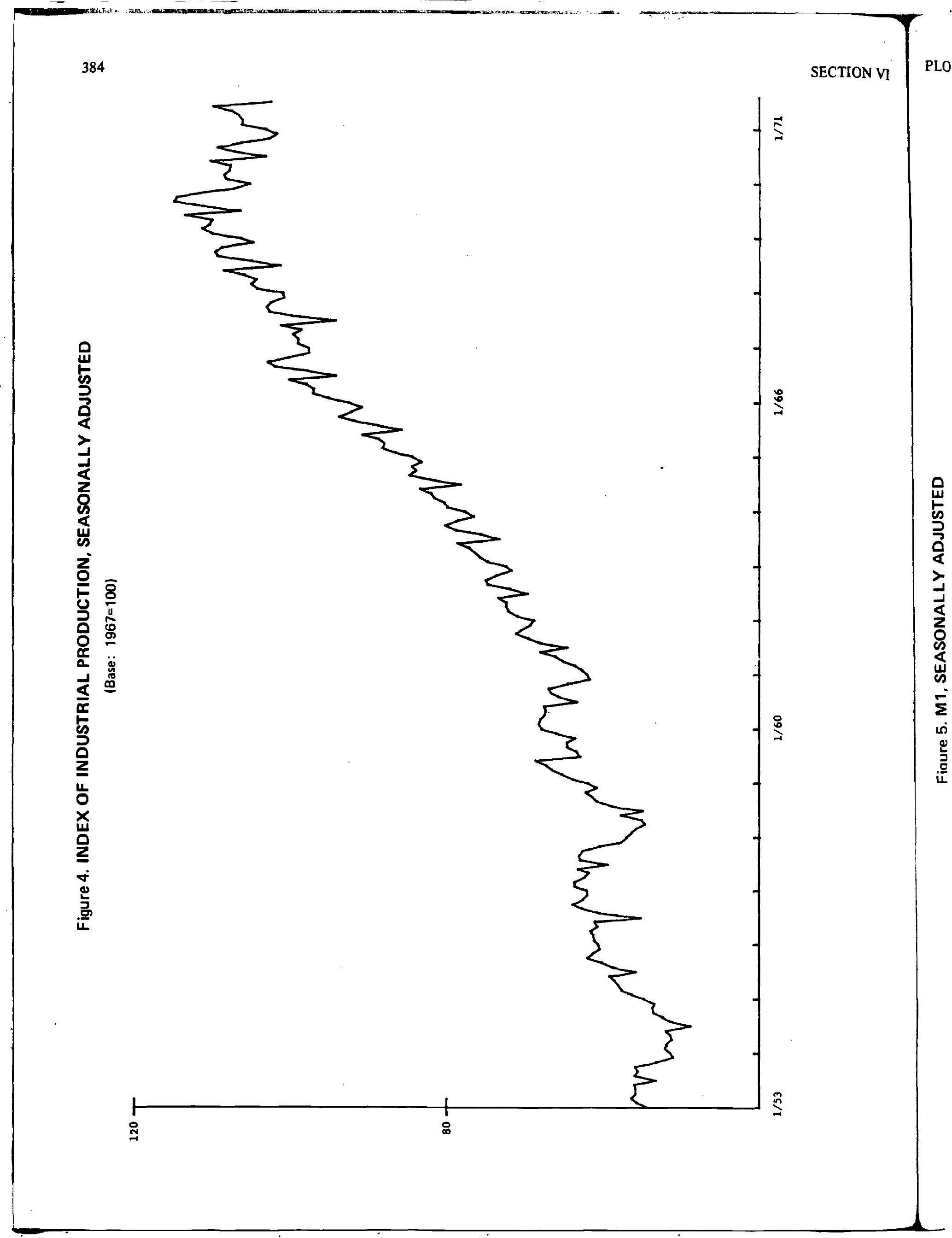





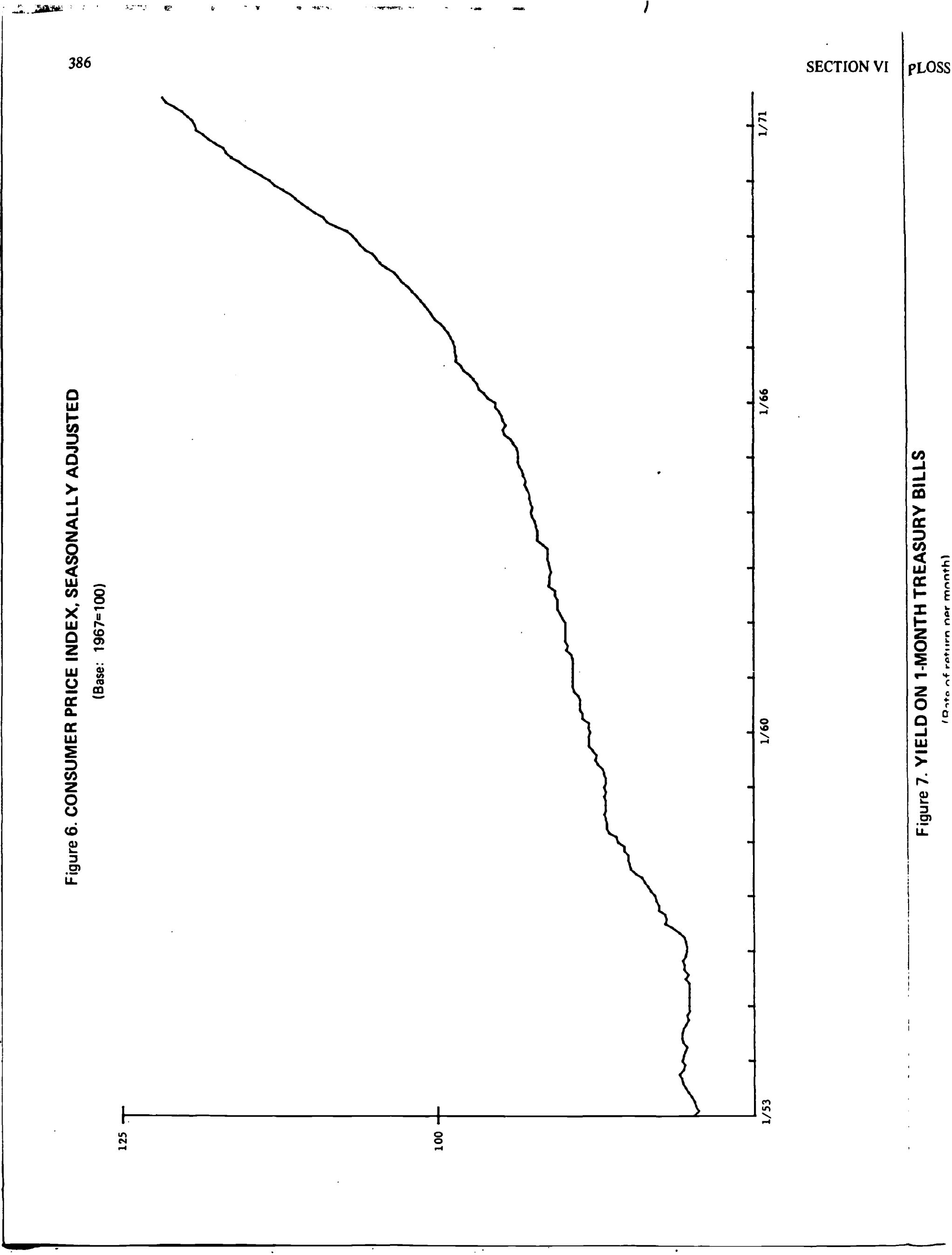




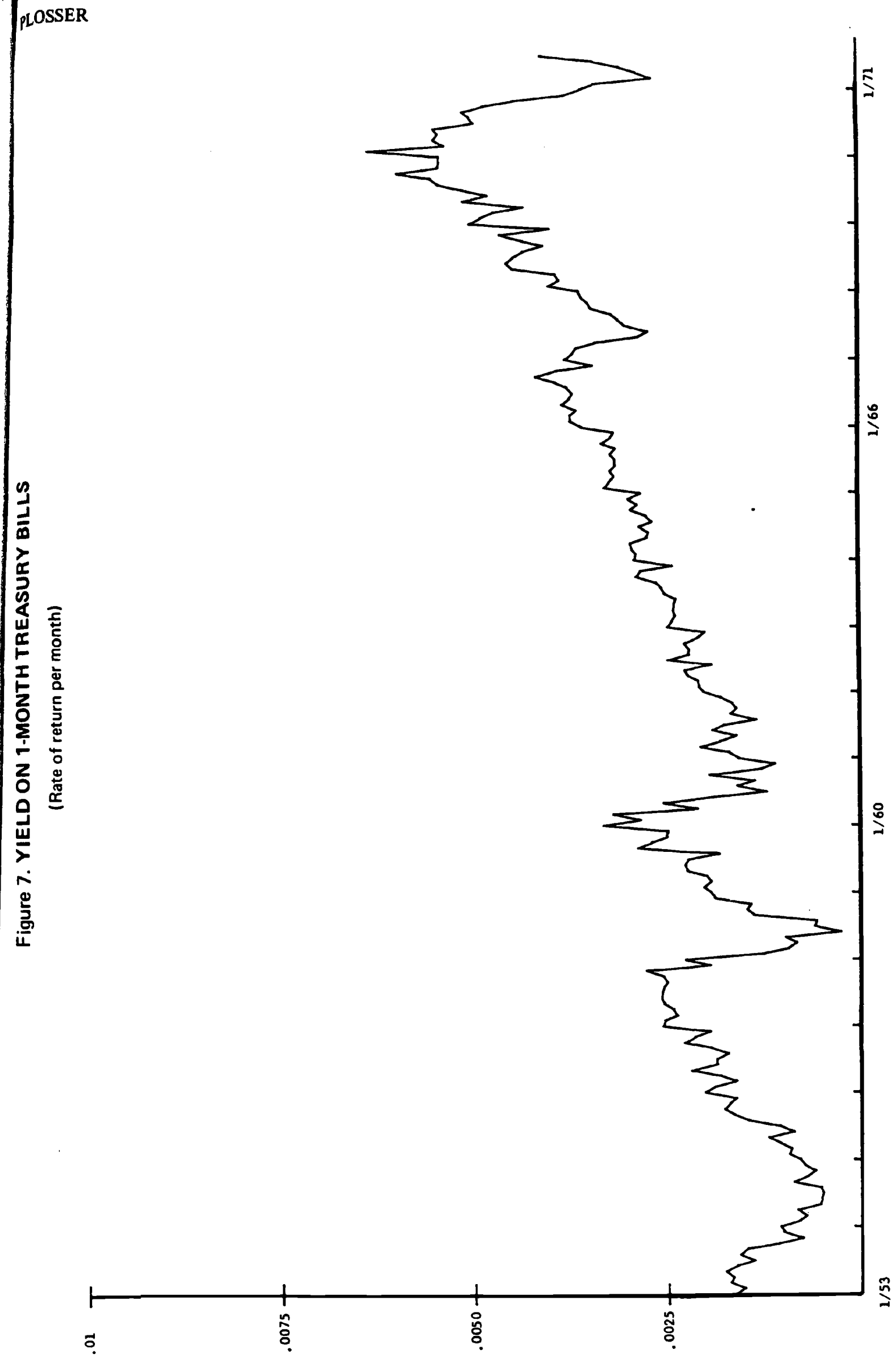


entering the FE. A likely possibility is a term that involves $u_{s k}$, the error from the process generating real output. This could occur, as was suggested in the section on the analysis of an economic model, if the base and real output were not independent. Another possibility is that $u_{2 t}$ is autocorrelated at low lags as well as at seasonal lags.

The second endogenous variable to be analyzed is the price level for which the CPI is used as representative. Recall, from the discussion in the section on the analysis of an economic model, that the analysis indicated that seasonal differences of the rate of inflation may very well appear as an AR process or even nonstationary in finite samples. Although an examination of the sample autocorrelation structure does not suggest nonstationarity, the results of fitting an ARIMA model to $\Delta_{12} r_{P_{t}}$ do point to that possibility. The model developed for this combination of differencing is a $(1,1,1)(0,1,1)_{12}$. The estimated values are presented in table 3 . Note that the AR parameter is very close to one suggesting nonstationarity. Unfortunately, as was indicated previously, the standard statistical tests cannot be performed here with satisfactory results. ${ }^{19}$ If the data are differenced, the preferred model appears to be $(0,2,1)(0,1,1)_{12}$. Since it is difficult to compare these models, the question of which one is preferred is left unanswered. However, the mere fact that this situation occurs lends support to the theory which suggested that such a phenomenon might exist.

The last of the endogenous variables is the nominal interest rate. The data used are the yields on 1-month Treasury bills. The same problem is experienced here that was experienced with the univariate model for prices. The theory suggests that either an AR model of the seasonal differences or even apparent nonstationarity of the seasonal differences might be observed in finite samples. It was seen that this was a result of the expectation mechanism at work. Note that, for the $(1,0,2)(0,1,1)_{12}$ model presented in table 3 ), the autoregressive coefficient is close to 1 . Once again, the standard testing procedures can not be utilized here when the null hypothesis is that the process is nonstationary. However, the model appears reasonably well behaved, showing no signs of redundancy or instability over time. Alternatively, the $(0,1,2)(0,1,1)_{12}$ also appears adequate, given the data.

The univariate time series models analyzed here display a remarkable degree of consistency, not only in the monthly process but in the seasonal process as well. The

\footnotetext{
${ }^{19}$ It is important to note that the AR and MA parameter appear close to each other, and, therefore, the possibility of redundancy must be considered. If the $(1,1,1)(0,1,1)_{12}$ model is redundant, then the parameters are not identified and the usual test procedures can not be utilized. However, a study of the standard errors of the parameter estimates and the correlation coefficient between them does not suggest redundancy or indicate that they are not identified.
}

actual models chosen are summarized in the following manner:

$$
\begin{aligned}
& \ln \left(B_{t}\right):(0,1,0)(0,1,1)_{12} \\
& \ln \left(Y_{t}\right):(0,1,2)(0,1,1)_{12} \\
& \ln \left(M_{t}\right):(0,1,3)(0,1,1)_{12} \\
& \ln \left(P_{t}\right):(0,2,1)(0,1,1)_{12} \text { or }(1,1,1)(0,1,1)_{12} \\
& i_{t}:(0,1,2)(0,1,1)_{12} \text { or }(1,0,2)(0,1,1)_{12}
\end{aligned}
$$

Though these parsimonious models should be interpreted as FE's with caution, the analyses indicate a reasonable amount of compatibility with the theory, as proposed in the section on the analysis of an economic model. If one were to rely solely on these results, several hypotheses about the structural model could not be rejected. For instance, since the AR portions of the exogenous variables are of degree zero, the AR portions of the endogenous variables should be of degree zero or greater. In fact, for prices and interest rates, the cases where the AR polynomials are greater than zero were exactly those that were suggested by the expectation mechanism. This speaks well for the hypothesis regarding rational expectations. Secondly, all of the models displayed a very similar seasonal moving average polynomial. The theory suggested that if $\Omega_{B}=\Omega_{Y}=\Delta_{12}$, then the seasonal polynomials for the endogenous variables could be factored out, and the seasonal multiplicative model would be the appropriate representation with a seasonal moving average polynomial close to $\Delta_{12}$. With the exception of the process for the money supply that has an SMA1 coefficient of 0.469 , the seasonal moving average parameter for the remaining endogenous variables was indeed close to $1(0.92$ for prices and 0.90 for the interest rate).

The results described in the previous paragraph should not be regarded as conclusive but suggestive of further analysis. The results of the transfer function analysis in the following subsection will provide further checks on the adequacy of the model as well as suggestions for possible modification.

\section{Analysis of the Transfer Functions}

The next step in the analysis of the model entails the estimation of the TF's. These distributed lag models, which express the current endogenous variables in the system in terms of current and past exogenous variables, are initially developed under the assumption that the model has been properly specified concerning which variables are endogenous and which are exogenous. However, this is not an innocuous assumption and should be considered an important part of the specification of any econometric model that should be checked against the data.

In this paper, use will be made of the cross-correlations between the residuals from the TF estimation and the various prewhitened exogenous variables. Under the null
PLC

hyp

cros

mea

sam

rent

ther

occl

spe

pro

mor

eco

non

the

$\alpha_{2}$

imf

out

sho

the

affe

1

On

hor

bas

ind

par

sea

by

tial

shc

MI

ind

the

suk

the

$d y_{1}$

In

mol

spe

\section{sh}

$\mathrm{mi}$

re:

ap

$\mathrm{pr}$.

$-$

sTr

frc

wt 
hypothesis that the model is correctly specified these cross-correlations are distributed independently with zero mean and large sample variance of $1 / T$, where $T$ is the sample size. If these cross-correlations between the current residual and future prewhitened inputs are nonzero, then the suspicion must be that feedback is likely to be occurring. This simple test should provide a check on the specification of the exogenous variables in the model. ${ }^{20}$

Table 4 reports the estimated TF's. ${ }^{21}$ These results provide additional checks on the specification of the model presented in the section on the analysis of an economic model. Based on the model, the TF for the nominal money stock is given by (45). Consequently, if the model is correctly specified, the structural coefficient $\alpha_{2}$ can be directly estimated. Also of interest is the implication on the error structure of (45). As was pointed out earlier, the error structure of the estimated model should display a seasonal moving average polynomial of the form $\left(1-L^{12}\right)$. However, this polynomial may be affected by seasonal autocorrelations in $u_{2 t}$.

The model presented in table 4 suggests an estimate of $\alpha_{2}$ of 0.172 with an estimated standard error of 0.053 . One would expect this parameter to be in the neighborhood of 1.0, i.e., the elasticity of $M 1$ with respect to the base should be close to 1 . Furthermore, the results indicate a significant amount of autocorrelation in $u_{2 z}$. In particular, as was concluded from the univariate model, seasonal autocorrelation seems to be present, as evidenced by the seasonal moving average parameter being substantially different from 1 . These results indicate that the base should not be considered the sole source of seasonality in M1.

Finally, the diagnostic checks applied to this model indicate evidence of nonzero cross-correlations between the current residuals and future values of the base. This suggests model misspecification in the sense that either there is feedback from M1 to the base or that there is a dynamic relationship between the multiplier and the base. In either case, the results provide evidence that the base is not the sole source of seasonality in $\mathrm{Ml}$ and that a more complicated money supply relationship needs to be specified.

Obviously, if the TF for $M 1$ is misspecified, the results should indicate that the other TF are also inadequate. In both the TF for prices and interest rates, the seasonal moving average parameter is less than 1. Furthermore, cross-correlation checks suggest that neither the base nor real income can be considered exogenous relative to prices. The source of this problem for the base is the appearance of $u_{2 t}$ in the error structure of the TF for prices. The source of the problem for real income is more

\footnotetext{
${ }^{20}$ See Haugh [8] and Plosser [17] for further discussion of this point.

${ }^{21}$ The observant reader may note that $\hat{\sigma}_{a}^{2}$ appears only slightly smaller and sometimes larger in the estimated TF's than $\hat{\sigma}_{u}^{2}$ obtained from the univariate models. This is due to the estimation techniques used. The univariate models are estimated using backforecasting, while the TF's are not. See Box and Jenkins [3] for a discussion.
}

likely a short-run Phillips curve relationship that is missing from the model. As for the interest rate equation, the cross-correlations indicate that the base is virtually independent of the nominal interest rate and that real income, though significantly related, shows evidence of feedback.

\section{Summary of Empirical Findings}

The results of the empirical analysis are not supportive of the economic model detailed in the section on the analysis of an economic model. The weaknesses of the model seem to stem from the seasonality in the unobserved money multiplier and its relationship with the monetary base and from the feedback effects indicating that real income is not an exogenous variable. These problems were clearly pointed out in the TF analysis but were suggested even in the analysis of the FE's. The FE's did not present enough evidence by themselves to reject the model, but the seasonal moving average parameter in the univariate model for M1, being much less than anticipated, was indicative of the weakness of the model's specification.

Certainly, at this point, the analyst attempting to construct a satisfactory seasonal model of the economy could proceed by modifying the model presented here in such a way as to eliminate the defects that are suggested by the analysis. For example, the inclusion of a real sector or a short-run Phillips curve would be a logical extension and would make output endogenous. In addition, further consideration must be given to the specification of the money supply relationship.

Despite the weakness of the model analyzed, it proved useful in demonstrating the methodological issues and the relevance of the form of analysis presented in the section on the methodology for analyzing seasonal economic models. Clearly, the techniques demonstrated here can be very useful in analyzing even very simple supply and demand models for any market.

\section{DISCUSSION}

In this paper, an effort has been made to investigate seasonality in economic data from a slightly different perspective than has been common in the literature. Interest is focused on demonstrating a methodology whereby seasonality can be directly incorporated into an econometric model. Utilizing this approach, one can determine what is implied about the seasonal properties of the endogenous variables in the model and use the implications to check the specification of the model against information in the data.

There are several different perspectives from which one can view the results of this approach. First, for the econometrician it has been shown how one might build seasonality into a model and check the specification. Although, in the example analyzed here, the seasonality entered through the exogenous economic variables, there are possibilities of including seasonal structural parame- 


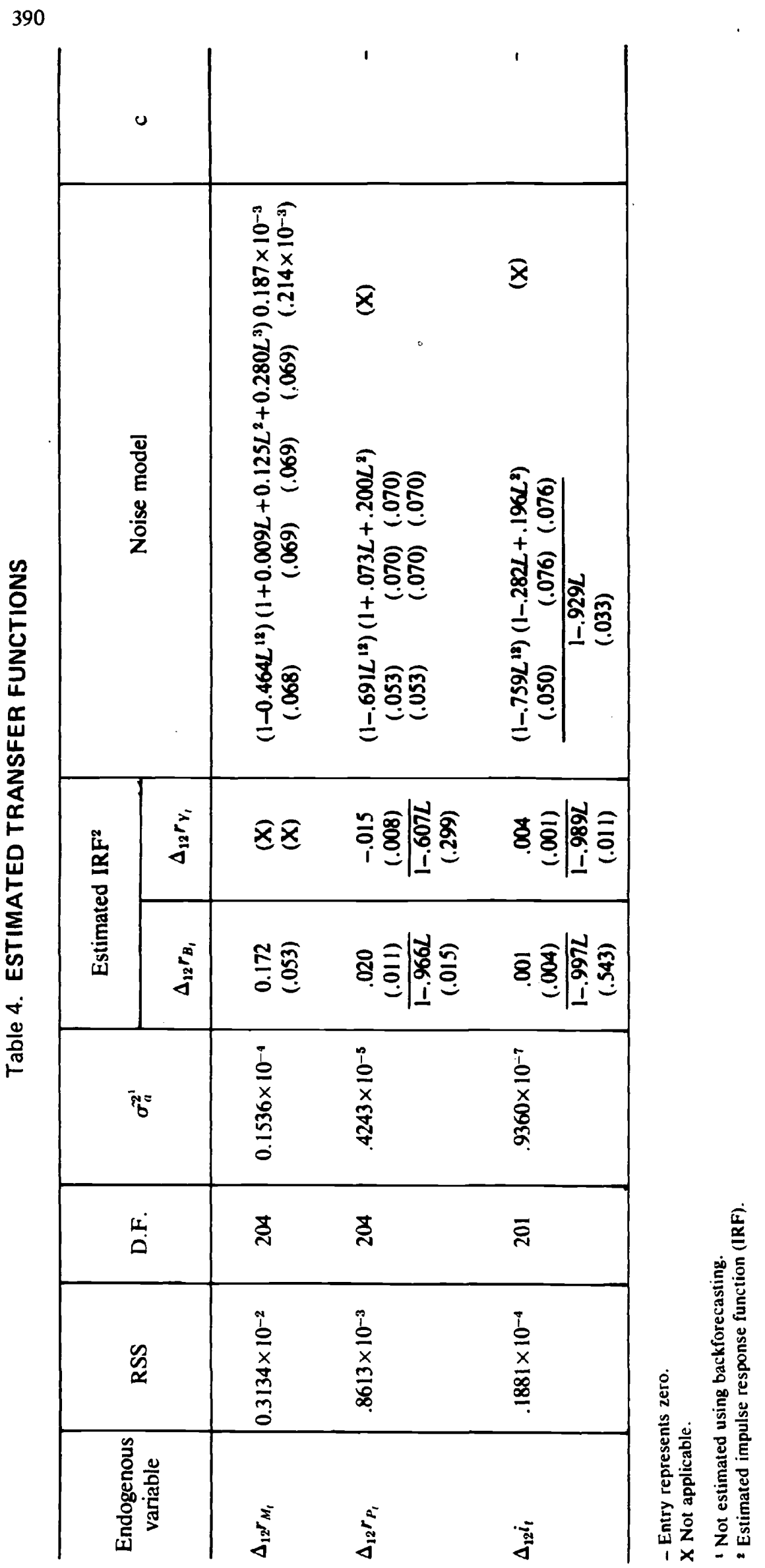


ters, or, in other types of markets, one may consider including stochastic seasonal variables, such as rainfall to act as the driving seasonal force. In proceeding in this manner, the model builder must carefully consider the possible sources of seasonal variation in his output variables. However, the analyst avoids using data that have been smoothed, using methods which may not be appropriate for his purposes.

There may still be reasons, however, for obtaining a seasonally adjusted series. In these cases, it would, of course, be ideal to make use of a model that would specify the source and type of seasonality. That is, through the use of an economic model and some economic analysis, understanding and insights can be gained concerning seasonal variation in economic time series, e.g., what might cause seasonality to change through time. Unfortunately, it may not be feasible to construct econometric models for every series that may need to be adjusted. Under these circumstances, obtaining a seasonally adjusted series based only on the past history of the series may prove to be necessary. However, it was demonstrated that, in general, the stochastic properties of economic variables in the system are not independent of the economic structure of which they are a part. Therefore, it would seem appropriate for those who wish to obtain adjusted series to study the stochastic behavior of the unadjusted data, investigating its form and properties, prior to adjustment. In making such an analysis, it is useful to realize that the standard linear econometric models do not imply that the multiplicative seasonal models of Box and Jenkins, in general, hold. Even in the case of the exogenous variables following the multiplicative specification, the endogenous variables would be a mixture of an additive and multiplicative process.

Many issues, of course, remain unresolved, but it would do no harm to suggest that econometricians or any consumer of economic data consider carefully the objectives of seasonal adjustment and why it is that adjusted data are desired. It may prove very easy to fail to see when one should stop making corrections and alterations to data once the process has started. Furthermore, the more the basic data are changed, the more cautiously any estimated relationship must be regarded, and, certainly, its results become more difficult to interpret. If some initial model does not provide an adequate explanation of the phenomenon under study, one should try to improve the model by explicit introduction of the factors that may have been omitted.

Seasonality, for instance, can be incorporated into an economic model in various ways. For example, it seems that the concern for abstracting from seasonality in economic data has stemmed partly from the belief that somehow economic agents respond to seasonal fluctuations differently from nonseasonal fluctuations, because these seasonal movements are so highly predictable. Therefore, using data which have not been adjusted may lead to misleading inferences about the true relationship, because the estimation would be contaminated by the relationship among the seasonal components. However, one can view this aspect of the seasonality problem as being part of a broader class of issues that has received a great deal of attention in the economics literature in the last few years. This literature is concerned with making the distinction between anticipated and unaticipated effects. It would seem a natural extension to merely consider seasonality as then belonging to this larger class of anticipated phenomenon. Similarly, from the standpoint of forecasting, one may only be concerned with whether a particular observation deviates from what might have been anticipated and whether or not the anticipated portion was seasonal or nonseasonal may be of less importance. 


\section{REFERENCES}

1. Anderson, T. W. "On the Asymptotic Distributions of Estimates of Parameters of Stochastic Difference Equations." Annals of Mathematical Statistics 30 (September 1959): 677-687.

2. - The Statistical Analysis of Time Series. New York: Wiley and Sons, 1971.

3. Box, G. E. P., and Jenkins, G. M. Time Series Analysis: Forecasting and Control. San Francisco: Holden-Day, Inc., 1970.

4. Box, G. E. P., and Pierce, D. A. "Distribution of Residual Autocorrelations in Autoregressive-Integrated-Moving Average Time Series Models." Journal of the American Statistical Association. (December 1970): 1509-1526.

5. Cleveland, W. P. "Analysis and Forecasting of Seasonal Time Series." Ph.D. dissertation, University of Wisconsin, 1972.

6. Dhrymes, P. J. Distributed Lags. San Francisco: Holden-Day, Inc., 1970.

7. Fama, E. F. "Short-Term Interest Rates as Predictors of Inflation." American Economic Review 65 (June 1975): 269-282.

8. Haugh, L. D. "The Identification of Time Series Interrelationships with Special Reference to Dynamic Regression Models." Ph.D. dissertation, University of Wisconsin, 1972.

9. Jorgenson, D. W. "Rational Distributed Lag Functions." Econometrica 34 (January 1966): 135-149.

10. Kmenta, J. Elements of Econometrics. New York: MacMillan Publishing Company, Inc., 1971.

11. Kuznets, S. Seasonal Variation in Industry and Trade. New York: National Bureau of Economic Research, 1933.

12. Laffer, A., and Ranson, D. " A Formal Model of the Economy." Journal of Business 44 (July 1971): 247 270.

13. Lucas, R. E. "Some International Evidence on Output-Inflation Tradeoffs," American Economic Review 63 (June 1973): 326-334.

14. Muth, J. F. "Rational Expectations and the Theory of Price Movements." Econometrica 29 (July 1961): 315-335.
15. Nelson, C. R. "The First Order Moving Average Process: Identification, Estimation, and Prediction." Journal of Econometrics 2 (July 1974): 121-142.

16. Pierce, D. A., and Mason, J. M. "On Estimating the Fundamental Dynamic Equations of Structural Econometric Models." Paper at the Econometric Society, New Orleans, La., 1971.

17. Plosser, C. I. "Time Series Analysis and Seasonality in Econometric Models with an Application to a Monetary Model." Ph.D. dissertation, University of Chicago, Graduate School of Business, 1976.

18. Plosser, C. I., and Schwert, G. W. "Regression Relationships Among Nonstationary Variables: Estimation and Inference in the Noninvertible Moving Average Process." Manuscript, University of Chicago, 1976.

19. Quenouille, M. H. The Analysis of Multiple Time Series. New York: Hafner Press, 1968.

20. Sargent, T. J. “' 'Rational' Expectations, the 'Real Rate of Interest' and the 'Natural Rate of Unemployment'." In Brookings Papers on Economic Activity, vol. 2. Edited by A. M. Okun and G. Perry. Washington: Brookings Institute, 1973.

21. U.S. Department of Commerce, Bureau of the Census. The X-11 Variant of the Census Method II Seasonal Adjustment Program, by Julius Shiskin, A. H. Young, and J. C. Musgrave. Technical Paper 15. Washington, D. C.: Goverment Printing Office, 1967.

22. U.S. Department of the Interior. "Economic Aspects of the Pacific Halibut Fishery," by J. Crutchfield and A. Zellner. In Fishery Industrial Research, April 1962.

23. Wallis, K. F. "Seasonal Adjustment and the Relations Between Variables." Journal of the American Statistical Association 69 (March 1974): 18-31.

24. White, J. S. "The Limiting Distribution of the Serial Correlation Coefficient in the Explosive Case." $A n$ nals of Mathematical Statistics 29 (December 1958): 1188-1197.

25. Zellner, A. "Time Series Analysis and Econometric Model Construction." In Applied Statistics. Edited by R. P. Gupta. Amsterdam: North-Holland, 1975, pp. 373-398. 
26. — and Palm, F. "Time Series Analysis and Simultaneous Equation Econometric Models." Journal of Econometrics 2 (May 1974): 17-54.
27. - "Time Series and Structural Analysis of Monetary Models of the U.S. Economy." Sankhya ser. C (June 1975): 12-56. 


\section{APPENDIX A}

This appendix details the derivations of the final equations (FE's) and the transfer functions (TF's) for the monetary model described in the subsection on model formulation. The analyses and interpretations of these results however, are primarily conducted in the text and not undertaken here.

The model is written as

$$
\begin{aligned}
r_{M_{t}}^{D} & =\alpha_{1} r_{Y_{t}}+\beta_{1} \Delta i_{t}+\gamma_{1} r_{P_{t}}+u_{1 t} \\
r_{M_{t}}^{S} & =\alpha_{2} r_{B_{t}}+u_{2 t} \\
i_{t} & =r_{P_{t+1}}^{*}+u_{3 t} \\
r_{P_{t+1}}^{*} & =E\left(r_{P_{t+1}} \cdot\right) \\
r_{M_{t}} & =r_{M_{t}}^{P_{t}}=r_{M_{t}}^{S}
\end{aligned}
$$

Substituting (A-5) and (A-3) into (A-1) results in

$$
r_{M_{t}}=\alpha_{1} r_{Y_{t}}+\beta_{1} \Delta\left(r_{P_{t+1}}^{*}+u_{3 t}\right)+\gamma_{1} r_{P_{t}}+u_{1 t}
$$

Combining this then with (A-2) and solving for $r_{P_{t}}$ yields

$$
\gamma_{1} r_{P_{t}}=\alpha_{2} r_{B_{t}}-\alpha_{1} r_{Y_{t}}-\beta_{1}\left(r p_{t+1}^{*}-r_{p_{t}}^{*}\right)-\beta_{1} \Delta u_{3 t}+u_{2 t}-u_{1 t}
$$

Now, for convenience, define

$$
X_{t} \equiv \alpha_{2} r_{B_{t}}-\alpha_{1} r_{Y_{t}}-\beta_{1} \Delta u_{3 t}+u_{2 t}-u_{1 t}
$$

and rewrite (A-7) as

$$
\gamma_{1} r_{P_{t}}=X_{t}-\beta_{1}\left(r_{P_{t+1}}^{*}-K_{P_{t}}^{*}\right)
$$

Under the assumption of rational expectations, the conditional expectation of $\boldsymbol{r}_{\boldsymbol{P}_{t+1}}$ can be calculated from (A-9).

$$
\underset{t}{E}\left(r_{P_{t+1}} \mid \cdot\right)=\gamma_{1}^{-1}\left[E X_{t+1}-\beta_{1} r_{P_{t+2}}^{*}+\beta_{1} r_{P_{t+1}}^{*}\right]
$$

or

$$
\left(\gamma_{1}-\beta_{1}\right) r_{P_{t+1}}^{*}=\underset{t}{E} X_{t+1}-\beta_{1} r_{P_{t+2}}^{*}
$$

The same can be done for $r_{P_{t+2}}$, yielding

$$
\left(\gamma_{1}-\beta_{1}\right) r_{P_{t+2}}^{*}=E X_{t+2}-\beta_{1} r_{P_{t+3}}^{*}
$$

which substituted into (A-11) gives

$$
\left(\gamma_{1}-\beta_{1}\right) r_{P_{t+1}}^{*}=E X_{t+1}-\frac{\beta_{1}}{\gamma_{1}-\beta_{1}} E X_{t+2}+\beta_{1} r_{P_{t+3}}^{*}
$$

Solving this recursively then yields

$$
r_{P_{t+1}}^{*}=\left(\gamma_{1}-\beta_{1}\right)^{-1} \sum_{j=0}^{\infty}\left(\frac{\beta_{1}}{\gamma_{1}-\beta_{1}}\right)^{j} E X_{t+j+1}
$$

Note that the anticipated rate of inflation is a weighted average of all expected future values of the exogenous variables. The weighting scheme itself is determined by the model or structure. The importance of these future values will, in large part, be determined by the term $\frac{-\beta_{1}}{\gamma_{1}-\beta_{1}}$

In order to generate expectations of future values of the exogenous variables, the assumption that they are ARIMA processes proves convenient. In fact, based on the analysis in the section on the methodology for analyzing seasonal economic models, one can interpret these processes as implying that these variables are generated by some unspecified structure and that the processes, used as inputs into this system, are merely the final equations from the model actually generating the monetary base and real income. These exogenous variables are then written as multiplicative seasonal time series models of the type described in the section on the methodology for analyzing seasonal economic models.

$$
\begin{aligned}
& \phi_{B}(L) \Gamma_{B}\left(L^{12}\right) \Delta_{12} r_{B_{t}}=\Theta_{B}(L) \Omega_{B}\left(L^{12}\right) u_{4 k} \\
& \phi_{Y}(L) \Gamma_{Y}\left(L^{12}\right) \Delta_{12} r_{Y_{t}}=\theta_{Y}(L) \Omega_{Y}\left(L^{12}\right) u_{5 t}
\end{aligned}
$$

Note that $\Gamma_{B}, \Gamma_{Y}, \Omega_{B}$, and $\Omega_{Y}$ represent the seasonal polynomials that have an explicit representation maintained to allow them to be traced through the model. Taking conditional expectations and dropping the $L$ 's for convenience produces

$$
\begin{aligned}
& \underset{t}{E r_{B_{t+1}}}=\Delta_{12}^{-1}\left[\left(1-\frac{\phi_{B} \Gamma_{B}}{\Theta_{B} \Omega_{B}}\right) \Delta_{12} r_{B_{t+1}}\right] \\
& \underset{t}{E r_{Y_{t+1}}}=\Delta_{12}^{-1}\left[\left(1-\frac{\phi_{Y} \Gamma_{Y}}{\theta_{Y} \Omega_{Y}}\right) \Delta_{12} r_{Y_{t+1}}\right]
\end{aligned}
$$

Equations (A-17) or (A-18) simply indicate that the onestep-ahead forecast can be written in terms of an infinite distributed lag of all past values where the weights are 
determined by the AR and MA polynomials. Through recursive calculations, one could obtain an expression for the expected value of any future observation, conditional on the information contained in the series at time $t$. That is,

$$
\begin{aligned}
& \underset{t}{E r_{B_{t+1}}}=\Delta_{12}^{-1} \pi j^{(B)}(L) \Delta_{12} r_{B_{t}} \\
& \left.\underset{t}{E r_{Y_{t+3}}}=\Delta_{12}^{-1} \pi\right\}^{n}(L) \Delta_{12} r_{Y_{t}}
\end{aligned}
$$

where $\pi f^{\cdot}(L)$ represents the polynomial in $L$ that gives weights applied to all previous observations of the exogenous variable to produce the minimum mean square error forecast for $j$ periods into the future. Note that the weights are a function of $j$, the forecast horizon.

Using (A-3), (A-14) can now be rewritten as

$$
\left(\gamma_{1}-\beta_{1}\right) i_{t}=\sum_{j=0}^{\infty}\left(\frac{-\beta_{1}}{\gamma_{1}-\beta_{1}}\right)^{j} E X_{t+j+1}
$$

or as

$$
\Phi \Delta_{12} i_{t}=\Psi_{1} \Delta_{12} r_{B_{t}}+\Psi_{2} \Delta_{12} r_{Y_{t}}+\Delta_{12} v_{t}
$$

where

$$
\begin{aligned}
& \Phi=\left(\gamma_{1}-\beta_{1}\right) \\
& \Psi_{1}=\alpha_{2} \sum_{j=0}^{\infty}\left(\frac{-\beta_{1}}{\gamma_{1}-\beta_{1}}\right)^{j} \pi j+1(L) \\
& \Psi_{2}=-\alpha_{1} \sum_{j=0}^{\infty}\left(\frac{-\beta_{1}}{\gamma_{1}-\beta_{1}}\right)^{j} \pi j+{ }_{1}(L) \\
& \Delta_{12} \nu_{t}=\Delta_{12} \sum_{j=0}^{\infty}\left(\frac{-\beta_{1}}{\gamma_{1}-\beta_{1}}\right)^{j} \cdot\left(E u_{2 t+j+1}-\beta_{1} E u_{t} t+j+1-E u_{1 t+j+1}\right) \\
& +\left(\gamma_{1}-\beta_{1}\right) \Delta_{12} u_{3 t}
\end{aligned}
$$

In matrix form, the system can now be written as

$$
\begin{gathered}
{\left[\begin{array}{ccc}
1 & -\gamma_{1} & -\beta_{1} \Delta \\
1 & 0 & 0 \\
0 & 0 & \Phi \Delta_{12}
\end{array}\right]\left[\begin{array}{c}
r_{M_{t}} \\
r_{P_{t}} \\
i_{t}
\end{array}\right]} \\
+\left[\begin{array}{cc}
0 & -\alpha_{1} \\
-\alpha_{2} & 0 \\
-\Psi_{1} \Delta_{12} & -\Psi_{2} \Delta_{12}
\end{array}\right]\left[\begin{array}{l}
r_{B_{t}} \\
r_{Y}
\end{array}\right]=\left[\begin{array}{l}
u_{1 t} \\
u_{2 t} \\
\Delta_{12} v_{t}
\end{array}\right]
\end{gathered}
$$

Referring to the first matrix on the left-hand side as $H_{11}$, its determinant can be written as

$$
\left|H_{11}\right|=\gamma_{1} \Phi \Delta_{12}
$$

while its adjoint is

$$
H_{11}^{*}=\left[\begin{array}{ccc}
0 & \gamma_{1} \Phi \Delta_{12} & 0 \\
-\Phi \Delta_{12} & \Phi \Delta_{12} & -\beta_{1} \Delta \\
0 & 0 & \gamma_{1}
\end{array}\right]
$$

By premultiplying both sides of (A-16) by $H_{11}^{-1}$ and then multiplying through by ${ }_{\text {, }}\left|H_{11}\right|$, the following can be obtained:

$$
\left|H_{11}\right|\left[\begin{array}{c}
r_{M_{t}} \\
r_{P_{t}} \\
i_{t}
\end{array}\right]=H_{11}^{*}\left[\begin{array}{cc}
0 & \alpha_{1} \\
\alpha_{2} & 0 \\
\Psi_{1} \Delta_{12} & \Psi_{2} \Delta_{12}
\end{array}\right]\left[\begin{array}{l}
r_{B_{t}} \\
r_{Y_{t}}
\end{array}\right]
$$

$$
+H_{11}^{*}\left[\begin{array}{c}
u_{1 t} \\
u_{2 t} \\
\Delta_{12} v_{t}
\end{array}\right]
$$

More explicitly,

$$
\begin{gathered}
\gamma_{1} \Phi \Delta_{12}\left[\begin{array}{c}
r_{M_{t}} \\
r_{P_{t}} \\
i_{t}
\end{array}\right]=\left[\begin{array}{cc}
\gamma_{1} \Phi \alpha_{2} & 0 \\
\Phi \alpha_{2}-\beta_{1} \Delta \Psi_{1} & -\Phi \alpha_{1}-\beta_{1} \Delta \Psi_{2} \\
\gamma_{1} \Psi_{1} & \gamma_{1} \Psi_{2}
\end{array}\right]\left[\begin{array}{c}
\Delta_{12} r_{B_{t}} \\
\Delta_{12} r_{Y_{t}}
\end{array}\right] \\
+\left[\begin{array}{ccc}
0 & \gamma_{1} \Phi \Delta_{12} & 0 \\
-\Phi \Delta_{12} & \Phi \Delta_{12} & -\beta_{1} \Delta \\
0 & 0 & \gamma_{1}
\end{array}\right]\left[\begin{array}{c}
u_{1 t} \\
u_{2 t} \\
\Delta_{12} v_{t}
\end{array}\right]
\end{gathered}
$$

The set of equations in (A-27) or (A-28) represent the set of TF's for the system. 
The set of FE can be obtained by substituting

$$
\begin{gathered}
{\left[\begin{array}{c}
\Delta_{12} r_{B_{1}} \\
\Delta_{12} r_{Y}
\end{array}\right]=\left[\begin{array}{cc}
\phi_{B} \Gamma_{B} & 0 \\
0 & \phi_{Y} \Gamma_{Y}
\end{array}\right]^{-1}\left[\begin{array}{cc}
\theta_{B} \Omega_{B} & 0 \\
0 & \theta_{Y} \Omega_{Y}
\end{array}\right]\left[\begin{array}{l}
u_{4 t} \\
u_{3 t}
\end{array}\right]} \\
=H_{22}^{-1} F_{22}\left[\begin{array}{l}
u_{4} \\
u_{3 E}
\end{array}\right]
\end{gathered}
$$

into (A-28), resulting in

$$
\begin{array}{r}
\left|H_{11}\right|\left|H_{22}\right|\left[\begin{array}{c}
r_{M_{t}} \\
r_{P_{t}} \\
i_{t}
\end{array}\right]=-H_{11}^{*} H_{12} H_{22}^{*} F_{22}\left[\begin{array}{l}
u_{4 t} \\
u_{3 t}
\end{array}\right] \\
+\left|H_{22}\right| H_{11}^{*}\left[\begin{array}{c}
u_{1 t} \\
u_{2 t} \\
\Delta_{12} v_{t}
\end{array}\right]
\end{array}
$$

or, more explicitly,

$$
\begin{aligned}
& \gamma_{1} \Phi \phi_{B} \phi_{Y} \Gamma_{B} \Gamma_{Y}\left[\begin{array}{c}
\Delta_{1}{ }_{m_{t}} \\
\Delta_{12} P_{t} \\
\Delta_{12} i_{t}
\end{array}\right] \\
& =\left[\begin{array}{cc}
\gamma_{1} \Phi \alpha_{2} & 0 \\
\Phi \alpha_{2}-\beta_{1} \Delta \Psi_{1}-\Phi \alpha_{1}-\beta_{1} \Delta \Psi_{1} \\
\gamma_{1} \Psi_{1} & \gamma_{1} \Psi_{2}
\end{array}\right]\left[\begin{array}{l}
\phi_{Y} \Gamma_{Y} \theta_{B} \Omega_{B} u_{u} \\
\phi_{B} \Gamma_{B} \theta_{Y} \Omega_{Y} u_{3}
\end{array}\right] \\
& +\phi_{B} \phi_{Y} \Gamma_{B} \Gamma_{Y}\left[\begin{array}{ccc}
0 & \gamma_{1} \Phi \Delta_{12} & 0 \\
-\Phi \Delta_{12} & \Phi \Delta_{12} & -\beta_{1} \Delta \\
0 & 0 & \gamma_{1}
\end{array}\right]\left[\begin{array}{c}
u_{1 t} \\
u_{2 t} \\
\Delta_{12} \nu_{l}
\end{array}\right]
\end{aligned}
$$

The derivations presented here are summarized and analyzed in the section on the analysis of an economic model.
1.

2.

3. 


\section{APPENDIX B}

Sources of Data

1. Monetary Base (B) data, seasonally unadjusted, were provided by the St. Louis Federal Reserve Bank. They are averages of daily figures and have been subject to no adjustment for changes in reserve margins or the like.

2. Index of Industrial Production (IIP) data were taken from the seasonally unadjusted, Federal Reserve Board Production Index, as specified by the Board of Governors of the Federal Reserve System Statistical Release G.12.3, "Business Indexes."

3. Money Stock (M1) data, not seasonally adjusted, are averages of daily figures for currency plus demand deposits, Board of Governors. of the Federal Reserve
System Statistical Release H.6, and the Federal Reserve Bulletin.

4. Consumer Price Index (CPI) data, not seasonally adjusted, were taken from the U.S. Department of Labor, Bureau of Labor Statistics.

5. Interest Rate (i) data was compiled by Fama [7] from Salomon Brothers quote sheets and represent the yields on 1-month U.S. Treasury bills.

All the above data, except for the monetary base and the yields on the Treasury bills, were taken from the data base as collected by Data Resources, Inc., as provided to the University of Chicago, Graduate School of Business, through the H. G. B. Alexander Research Foundation. 


\title{
COMMENTS ON "A TIME SERIES ANALYSIS OF SEASONALITY IN ECONOMETRIC MODELS" BY CHARLES I. PLOSSER
}

\author{
Gregory C. Chow \\ Princeton University
}

The conference participants can probably be divided into two groups: Those who believe in using a specific model because they think they have a good one, and those who prefer not to choose a model but rather to devise robust methods. Time does not allow me to discuss these opposing positions. However, I agree with the late Chairman Mao that we should let a hundred flowers bloom.

Among those who believe in using a model, some would perform multivariate, and others would perform univariate time series analyses. The papers in this session are devoted to multivariate time series analyses. In particular, the basic proposition of Charles Plosser's paper [3] is that the analysis of seasonal fluctuations in economic time series, and the construction of econometric models can be made an integrated process. My comments will be divided into three parts. First, I will summarize the main features of Plosser's particular approach to combine a seasonal model with a traditional simultaneous econometric model. Second, I will comment on the specific illustrative example used in his paper. Third, I will suggest an alternative approach to integrate seasonal fluctuations with a simultaneous-equations model.

In Plosser's approach, it is first assumed that the economic model for the endogenous variables $y_{l}$ and the exogenous variables $x_{t}$ can be written as

$$
\begin{aligned}
H_{11}(L) y_{t}+H_{12}(L) x_{t} & =F_{11}(L) e_{1 t} \\
H_{22}(L) x_{t} & =F_{22}(L) e_{2 t}
\end{aligned}
$$

where $H_{i j}$ and $F_{i j}$ are polynomials in the lag operator $L$ with matrix coefficients, and $e_{1 t}$ and $e_{2 t}$ are serially independent random variables. Second, there exist seasonalities in $x_{t}$ that can be described by the multiplicative seasonal time series models of Box and Jenkins [1], i.e., $H_{22}(L)$ and $F_{22}(L)$ take the special form, such that the ith element $x_{t}^{i}$ of $x_{t}$ is determined by

$$
\Gamma\left(L^{s}\right) \phi(L)\left(1-L^{s}\right)^{D}(1-L)^{d} x_{t}^{i}=\Omega\left(L^{s}\right) \Theta(L) e_{2 t}^{i}
$$

where $s=12$ if seasonal fluctuations in monthly data are being studied, the operators $\left(1-L^{g}\right)^{D}$ and $(1-L)^{a}$ will serve to difference the original series seasonally and consecutively, $\phi(L)$ and $\Theta(L)$ are the usual autoregressive and moving-average operators for the process governing $x_{i}^{i}$, and, finally, $\Gamma\left(L^{s}\right)$ and $\Omega\left(L^{s}\right)$ are seasonal $A R$ and MA 398 polynomials (or polynomials in $L^{12}$ ) that help characterize the seasonalities in the process. Strictly speaking, all operators in (2) should be superscripted by the index $i$ for the ith exogenous variable, but this superscript has been omitted. The basic approach is to trace the implications of the particular specifications of $\Gamma^{y}\left(L^{s}\right)$ and $\Omega^{i}\left(L^{8}\right)$, which are parts of the specifications of $H_{22}(L)$ and $F_{22}(L)$, respectively, on the transfer functions and the final equations of the model (1a) and (lb), thus imposing restrictions on the latter equations for statistical analysis and testing.

The transfer functions, often called the final form of an econometric model (to be distinguished from the final equations, which will be presented) are obtained by using (1a) to express $y_{t}$ as a distributed lag function of $x_{t}$ and $e_{1 t}$, i.e.,

$$
y_{t}=-H_{11}^{-1} H_{12} x_{t}+H_{11}^{-1} F_{11} e_{1 t}
$$

The final equations are obtained by using the identity $H_{11}^{-1} \equiv\left|H_{11}\right|^{-1} H_{11}^{*}, H_{11}^{*}$ being the adjoint matrix of $H_{11}$ and $\left|H_{11}\right|$ being its determinant, to isolate a (common) scalar autoregressive polynomial in $L,\left|H_{11}(L)\right|$, for each of the elements of $y_{t}$, rather than the original matrix autoregressive polynomial $H_{11}(L)$ for the vector $y_{t}$

$$
\left|H_{11}\right| y_{t}=-H_{11}^{*} H_{12} x_{t}+H_{11}^{*} F_{11} e_{1 \ell}
$$

Using $x_{t}=H_{22}^{-1} F_{22} e_{2 t}=\left|H_{22}\right|^{-1} H_{22}^{*} F_{22} e_{2 t}$ to substitute for $x_{t}$ in (4), we obtain

$$
\left|H_{11}\right|\left|H_{22}\right| y_{t}=-H_{11}^{*} H_{12} H_{22}^{*} F_{22} e_{2 l}+\left|H_{22}\right| H_{11}^{*} F_{11} e_{11}
$$

Insofar as the specifications of the seasonal AR and MA polynomials $\Gamma^{i}\left(L^{s}\right)$ and $\Omega^{i}\left(L^{s}\right)$ affect $H_{22}$ and $F_{22}$, respectively, they also impose restrictions, through $\left|H_{22}\right|, H_{22}^{*}$, and $F_{22}$ on the final equations (5), and these restrictions can be confronted with the time series data on the endogenous variables.

Having briefly described the general approach, let me list its major characteristics as follows:

1. The linear simultaneous equations model (1a) explains the observed time series $y_{t}$ by the observed $x_{t}$, rather than the seasonally adjusted series, as might be supposed.

2. Seasonality in $y_{t}$ is explained solely by the seasonality in $x_{t}$ and not by seasonality in the parameters or other mechanisms. 
3. Seasonality in $x_{t}$ is described by the multiplicative seasonal model of Box and Jenkins for each exogenous variable separately and not by a simultaneous multiplicative seasonal model for the vector $x_{t}$, nor by some additive model.

4. The algebraic relationships between the specifications of the seasonal variations in the exogenous variables such as imbedded in $H_{22}(L)$ and $F_{22}(L)$ through equation (2) and the final equations (5), appear to be very complicated. Even for medium size models, it appears difficult to trace explicitly the algebraic implications of the seasonal equations (2) on the final equations (5). Thus, the approach of this paper might be applicable only to very small and very specialized models.

5. In general, the transfer functions (3) and even the partially final equations (4), where $x_{t}$ has not been eliminated, do not involve $H_{22}(L)$ and $F_{22}(L)$ and are, therefore, not affected by the specifications of the seasonal pattern for $x_{t}$. It is only in the special example, treated by Plosser, that the seasonalities in $x_{t}$ impose restrictions on the transfer functions. The reason is the rational expectations hypothesis adopted in the illustrative example. By this hypothesis, some endogenous variables will depend on the conditional expectations of $x_{t}$ which, in turn, are distributed lag functions of past $x_{t-k}$ as implied by the seasonal model (2). Hence, the relations between $y_{t}$ and the lagged exogenous variables, as given by the transfer functions, are also restricted by the specification of the seasonal pattern given by equation (2).

6. The approach does not yield a decomposition of an economic time series into seasonal, trend-cycle, and irregular components. Purely for the purpose of measuring the changes in such important economic variables such as industrial production, the consumer price index, and the rate of unemployment net of seasonal effects, the approach fails to provide an answer.

Concerning the illustrative example, the author is aware of many of its limitations and shortcomings. I will, however, emphasize several problems with this example, since they have bearing on the general applicability of the proposed approach. The simple model explains three endogenous variables-money stock, a general price index, and the rate of interest-by two exogenous variables-the monetary base and the agggregate real outputto be measured by the Index of Industrial Production. The first problem concerns the use of the selected exogenous variables alone to account for the seasonalities in the econometric model. The first exogenous variable, the monetary base, is a policy instrument. Why should the monetary authorities determine the monetary base following a certain seasonal pattern that is independent of all the endogenous variables in the economy? There is a general problem of attributing seasonalities in the policy variables that are independent of the internal workings of the economy. Are not the increases in the demand for money during certain seasons, such as the Christmas-New Year holidays, due to the seasonal pattern of demand itself? Similarly, are not seasonal fluctuations in the Index of Industrial Production due, at least partly, also to the seasonal pattern of demand? It seems difficult to attribute all the seasonalities in any reasonable econometric model to the exogenous variables, as usually defined, without including at least some seasonal dummy variables that are not used by the author.

The second problem is that the mechanism transmitting the seasonalities in the exogenous variables to the endogenous variables, such as the first transfer function relating the changes in the money stock to the changes in the monetary base in the general formulation of table 1 , is far too simple to be useful. It is likely that such simple formulations are used in order to keep track of the algebraic relations between the transfer functions and the final equations, on the one hand, and the seasonal specifications of the exogenous variables on the other. This example raises doubt on whether more complicated formulations can be explicitly analyzed by the approach of this paper. Third, even in this oversimplified example, analysis and interpretation of the implications of the seasonalities in $x_{t}$ on the dynamic characteristics of $y_{t}$ have encountered difficulties, as exemplified by the final equation (50) for the rate of change in the price level. Fourth, related to the third problem is the difficulty of obtaining conclusive results from statistical analysis of the final equations. The final equations are derived from the many characteristics of the econometric model besides the seasonal characteristics of the exogenous variables. To attribute the dynamic characteristics of the final equations to the seasonal specifications alone would, therefore, be extremely difficult. Could not the same dynamic implications for the final equations have been derived from a different seasonal model combined with different specifications for the remaining parts of the econometric model? This problem would surely be more serious for larger models. Fifth, one may question whether the particular method of trend elimination by way of various differencing operations is adequate. The requirement appears to exist that, after the differencing operations, the resulting model should have autoregressive polynomials having roots which will insure that the time series are stationary. How much does one sacrifice by restricting the method of trend elimination to differencing operations and by insisting that only stationary models be studied? Sixth, few significant economic conclusions seem to have resulted from the example.

As the above comments may apply not only to the specific economic example, one would question the general applicability of the method proposed. There is no question that this paper has suggested interesting ideas and methods for analyzing seasonalities in economic time series. However, unless these problems could be resolved and a serious and relevant economic application could be provided to demonstrate its usefulness, I am afraid that 
the approach would not be widely accepted by analysts of seasonalities in economic time series.

By way of providing an alternative approach to combining seasonal analysis with the construction of an econometric model for cyclical fluctuations, I would like to suggest the following specifications and methods of analysis. In his note, Adrian Pagan [2] has pointed out the possibility of applying the filtering and estimation methods for state-space models to the estimation of seasonal and cyclical components in economic time series. The following suggestion is essentially a combination of an econometric model for the cyclical components with the filtering and estimation of the seasonal components formulated in a state-space form. Assume, first, that the vector $y_{t}$ of endogenous variables is the sum of cyclical, seasonal, and irregular components, as given by

$$
y_{t}=y_{t}^{c}+y_{t}^{s}+v_{t}
$$

and, second, that the cyclical component $y_{t}^{c}$ is governed by the following model:

$$
y_{t}^{c}=A y_{t-1}^{c}+C x_{t}+b+u_{t}
$$

where $x_{t}$ is a vector of exogenous variables and $u_{t}$ is a vector of random disturbances. The exogenous variables might or might not be seasonally adjusted, but this issue does not affect our analysis, since the vector $x_{t}$, seasonally adjusted or not, is treated as predetermined. Third, an autoregressive seasonal model is assumed for the seasonal component, as illustrated by, but not confined to, the simple scheme

$$
y_{t}^{s}=B y_{t-12}^{s}+w_{t}
$$

where $w_{t}$ consists of random residuals. Combining equations (7) and (8), we can write the vector $z_{t}$ of unobserved components in the form

$$
z_{t}=M z_{t-1}+N x_{t}+\epsilon_{t}
$$

where $z_{t}$ includes both $y_{t}^{c}$ and $y_{t}^{s}$ as its first two subvectors as well as the necessary lagged $y_{i-k}^{c}$ and $y_{i-k}^{s}$ to transform the original model (7) of possibly higher order and equation (8) of order 12 into first order, the matrix $M$ will depend on the matrices $A$ and $B$, the matrix $N$ will depend on $C$ and $b$, the vector $x_{t}$ will include dummy variables to absorb the intercept $b$ of equation (7), and $\epsilon_{t}$ will depend on $u_{t}$ and $w_{t}$. Equation (6) can be rewritten as

$$
y_{t}=\left[\begin{array}{lll}
l & 1 & 0
\end{array}\right] z_{t}+v_{t}
$$

Thus, equations (9) and (10) are in the standard statespace form, the first explaining the unobserved state variables $z_{t}$ and the second relating the observed $y_{t}$ to $z_{t}$. Given observations on $y_{t}$ and $x_{t}$, the conditional expectations of the unobserved components of $z_{t}$ can be estimated by the well-known techniques of Kalman filtering and smoothing, provided that the parameters $A, C, b$, and thus $M$ and $N$ are known.

In practice, the parameters $A, C$, and $b$ of the econometric model (7) are unknown. One can employ seasonally adjusted data for $y_{i}^{c}$, obtained by a standard seasonal adjustment procedure, and the standard statistical estimation techniques to obtain estimates of $A, C$, and $b$. Using these estimates, one can then compute estimates of the seasonal and cyclical components in $z_{t}$ by Kalman filtering and smoothing. The new estimates of $y_{i}^{c}$ will serve as new data for the reestimation of the econometric model (7). New estimates of the seasonal components $y_{i}^{s}$ will result from this process. I believe that this approach, as well as the approach suggested by Plosser to combine econometric modeling with the specifications of seasonalities in economic variables, should be further studied and pursued. In closing, I would like to thank and congratulate Charles Plosser for having provided us with an interesting, original, and thought-provoking paper. 


\section{REFERENCES}

1. Box, George E. P., and Jenkins, G. M. Time Series Analysis, Forecasting, and Control. San Francisco: Holden-Day, Inc., 1970.

2. Pagan, Adrian. "A Note on the Extraction of Compo- nents from Time Series." Econometrica 43 (January 1975): $163-168$.

3. Plosser, Charles I. "A Time Series Analysis of Seasonality in Econometric Models." Included in this report. 


\title{
COMMENTS ON "A TIME SERIES ANALYSIS OF SEASONALITY IN ECONOMETRIC MODELS” BY CHARLES I. PLOSSER AND THE IMPLICATIONS FOR POLICYMAKERS AND MODELBUILDERS
}

\author{
Raymond E. Lombra \\ Pennsylvania State University
}

In the preceding paper, Charles Plosser has presented an interesting alternative procedure for dealing with seasonality in econometric models. The fundamental premise he argues is that "economic concepts may be useful in arriving at a better understànding of seasonality. Within the context of an economic structure ... the seasonal variation in one set of variables ... should have implications for the seasonal variation in closely related variables" [4]. In general, this view, articulated by Nerlove [3, p. 263] and others some time ago, leads one to look beyond the mechanical approaches for dealing with seasonality, such as the Census X-11 procedure, and instead seek a structural approach. As is well known, the major problems with the mechanical approaches revolve around defining and obtaining an optimal decomposition of the unobserved seasonal component from the observed series. On the other hand, the major difficulty associated with a structural approach concerns the identification of the correct structure. I suspect many of us would agree that the structural approach is preferable. However, the difficulties associated with making such an approach operational have led most producers and consumers of adjusted data to adopt the mechanical approaches as a kind of second-best solution. ${ }^{1}$

No doubt, nearly all of our empirical work suffers from problems generated by using imperfectly adjusted data. However, the major issues revolve around the seriousness of such problems and whether or not an alternative method for dealing with seasonality, such as Plosser's, can provide us with a better understanding of the processes generating seasonality. If the latter can be accomplished, it may assist the producers of seasonally adjusted data in improving their procedures and, thereby, aid the users of such data.

Since the technique developed in Plosser's approach is applied to a simple monetary model of the U.S. economy,

\footnotetext{
'It should be pointed out that producers and consumers of data probably have different objective functions and face different constraints. A producer like the Census Bureau must turn out a huge number of series on a timely basis. This being the case, a structural approach may only be useful as a diagnostic tool, employed from time to time, to evaluate the output from a mechanical approach. Researchers, on the other hand, desire to minimize the distortions that seasonal fluctuations can generate in trying to identify longer run relationships. Plosser's paper is primarily directed at producing a technique applicable to the latter set of problems.
}

it might be useful to illustrate the type of data problems faced by the Federal Reserve System, the key user of money stock data in the United States. In a recent paper, Fry [8] applied a variety of seasonal adjustment techniques to monthly money sfock data. In general, he found that "a variety of plausible seasonal methods produce roughly similar turning points in the M1 series, but seasonally adjusted growth rates differ substantially in the short run" [8, pp. 1-2]. The following table is part of a larger table in Fry's paper $[8, p$. 14]. It shows the range of M1 growth

\section{Table 1. RANGE OF M1 GROWTH RATES YIELDED BY ALTERNATIVE ADJUSTMENT PROCEDURES}

(Growth rates are expressed as seasonally adjusted annual percentage rates)

\begin{tabular}{|c|c|c|c|}
\hline 1975 & High & Low & Range \\
\hline January . . . . . . . & -2.1 & -10.9 & 8.8 \\
\hline February ........ & 4.3 & -6.4 & 10.7 \\
\hline March $\ldots \ldots \ldots$ & 11.5 & 7.2 & 4.3 \\
\hline April .......... & 8.0 & 1.3 & 6.7 \\
\hline May $\ldots \ldots \ldots$ & 11.3 & 5.5 & 5.8 \\
\hline June ......... & 19.2 & 11.7 & 7.5 \\
\hline July ...... & 6.2 & 2.9 & 3.3 \\
\hline August $\ldots \ldots$ & 7.0 & 1.2 & 5.8 \\
\hline September . . & 6.1 & .4 & 5.7 \\
\hline October .... & .4 & -3.3 & 3.7 \\
\hline November .... & 13.1 & 3.2 & 9.9 \\
\hline December ....... & 2.8 & -6.9 & 9.7 \\
\hline Average .... & $(X)$ & $(\mathrm{X})$ & 6.8 \\
\hline
\end{tabular}

X Not applicable.

Raymond E. Lombra, an associate professor of economics, Pennsylvania State University. The author expresses his gratitude to Herbert Kaufman and Dennis Farley for helpful comments during the preparation of this paper. 
rates for 1975 , produced by applying 11 different seasonal adjustment procedures to the unadjusted data. ${ }^{2}$

As can be seen, the average range of monthly growth rates, produced by the 11 procedures, was nearly 7 percentage points (or $\$ 1.7$ billion). Perhaps I am overly sensitive to these results, but, in view of the fact that the short-run target ranges for M1 specified by the Federal Open Market Committee are typically 4 percentage points wide, it is a bit unsettling to learn that the implied confidence interval for the adjusted data is so wide. ${ }^{3}$

Another serious problem for the Federal Reserve System concems the ex post facto revisions in the seasonal factors that are initially adopted. As is well known, the factors derived from an X-11-type procedure used to adjust current data (which, in effect, are forecasts of seasonal factors) will be subject to revision in following years as the extrapolations of the terminal years in the ratios and moving averages are replaced with actual data. This procedure along with the way outliers are handled often result in significant ex post facto revisions in the date relative to the data policymakers initially had available to guide their actions. The following figure vividly illustrates this problem. The first published data for the money stock are often very different from the revised data, and the revised data tend to show considerably less variance. It seems clear that the variety of issues underlying these adjustment problems firmly establish the need for new approaches to seasonal analysis.

The primary focus of Plosser's paper is to build an econometric model that contains an explicit specification of the causal sequence generating seasonality in the endogenous variables. More specifically, the central hypothesis to be tested is that seasonality enters the system through the processes generating the exogenous variables. The presumption is that the structure then transmits the exogenous seasonal impulses to the endogenous variables. An alternative hypothesis, as Plosser recognizes, is that various parameters in the structure could fluctuate seasonally. The result, of course, would be observed seasonality in the endogenous variables without any seasonality in the exogenous variables. The latter hypothesis would imply a different model for each season and would be considerably more difficult to specify and estimate.

The simple macromodel constructed to test the hypothesis treats the monetary base and real income as exgenous, and, therefore, it is assumed that seasonality enters the system only through systematic movements in these variables. In addition, the real rate of interest is, in effect, also treated exogenously, since a constant expected value is assumed.

\footnotetext{
2 The 11 procedures included various $\mathrm{X}-11$ options (multiplicative, additive, movng seasonal, and constant seasonal), multiplicative and additive versions of a regression technique, developed by Stephenson and Farr [6], and a new daily method, developed by Pierce, VanPeski, and Fry [9].

${ }^{3}$ See [2] for a detailed discussion of the role of money stock target ranges in the strategy of monetary policy.
}

As is usually true in applied econometrics, there are a variety of concessions that a researcher must make to translate a theoretical construct into a model that can be estimated. This being the case, it is often easy to critique the compromises made in doing empirical work. Of course, there are a variety of such compromises that Plosser has made. Rather than trace the problems with the model in detail, it is sufficient to point out that, when Plosser checked his empirical findings against the restrictions implied by the model and accompanying assumptions, he found the model and assumptions deficient. More specifically, there appeared to be evidence of seasonality in the structure (particularly the money multiplier), and the assumed exogeniety of real income and the monetary base appeared to be inappropriate. Although the results are, in some sense, negative, they do reveal the major strength of Plosser's approach: By constructing a model with an explicit specification of the processes generating seasonality, various restrictions on the model were imposed and could be checked. This diagnostic checking, in turn, will lead to improved model specification.

Unfortunately, it would appear that modelbuilders rarely check their results for the effects of seasonality and for sensitivity to alternative seasonal adjustment procedures for the input data. This void in hypothesis testing has become potentially more serious with the development of monthly and weekly models. Assuming policymaker performance, in the short-run, is dependent ultimately on the reliability of short-run data and the robustness of such models, the potential costs of poor seasonal analysis are obvious.

The results in Plosser's paper probably come as no great surprise to many of us. For example, if the description of monetary policy in (2) is reasonably accurate, it seems fairly clear that, in the short run, movements in the monetary base are not exogenous, but rather, are a function in part of contemporaneous movements in income and interest rates. ${ }^{4}$ This being the case, correctly explaining the seasonality in the base will require the specification of a reaction function for the Federal Reserve System that captures seasonal objectives. ${ }^{5}$

More generally, the systematic movement (in contrast to the strictly seasonal movements) in the money stock over time are the product of natural seasonals on the demand side (such as seasonal movements in the currency to demand deposit ratio) and the supply side (i.e., seasonal aspects of bank behavior), and the systematic movements in policy as reflected in the variance of the monetary

\footnotetext{
4o illustrate, the correlation coefficient between the monthly seasonal factors estimated by $X-11$ for total reserves (a critical part of the monetary base) and the 90-day Treasury-bill rate for 1960-1975 is about 0.6 .

'The seasonal forces the Federal Reserve System may be concerned with might include, i.e., regular Treasury financings (such as quarterly refundings) and the increase in money demand over the last half of the year as production, consumption, and borrowing rise in anticipation of Christmas.
} 


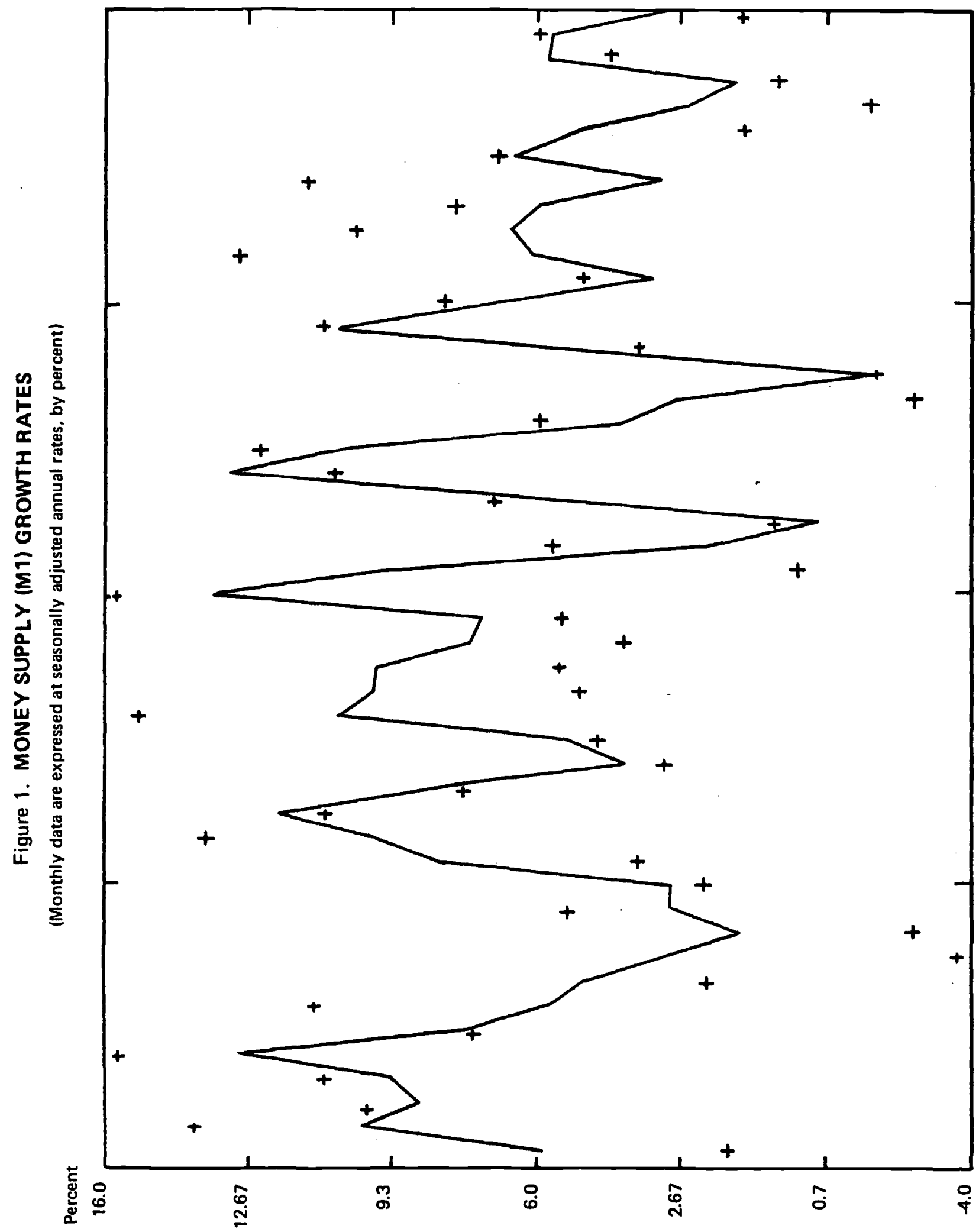


base. However, the systematic movements in the base may reflect both cyclical and seasonal phenomena. ${ }^{6}$

To illustrate, assume that there is a cyclical upswing in economic activity which lasts 18 months (January of year 1 through June of year 2) and that the Federal Reserve System allows the base and, therefore, the money stock to expand as much as is necessary to hold short-term interest rates constant throughout the period. Subsequently, we will observe systematic movement in the money stock in the January-June period of both years, and I would guess that few would want to characterize such systematic movement as seasonal. Against this background, I would expect some systematic movement to remain in the $M 1$ series, even after perfect seasonal filtering.

In summary, I would want to reserve judgment on the ultimate payoff of Plosser's time series approach in this specific area until it can be shown that a considerably more complex model can, in fact, be successfully estimated. However, on the other hand, Plosser has clearly demonstrated the need for modelbuilders to consider

${ }^{-}$This issue is discussed in detail in [1] and in the recent report of the Advisory Committee on Monetary Statistics [7]. carefully the souces of seasonal variation in the endogenous variables, and he has developed a general method to identify the sources that appear feasible. As he states, "the stochastic properties of economic variables in the system are not independent of the economic structure of which they are a part. Therefore, it would seem appropriate for those who wish to obtain adjusted series to study the stochastic behavior of the unadjusted data, investigating its form and properties prior to adjustment" [4].'

Finally, Plosser's work along with several other recent papers $[1 ; 5 ; 10]$ amply demonstrate that various estimated relationships can be quite sensitive to the way seasonality is handled. My own guess is that such problems will ultimately be solved only when talented teams of researchers (econometricians, theoreticians, and institutionalists), like those that combined to build the large macromodels, can be brought together to extend the work of Plosser and others on the structure of seasonality. ${ }^{8}$

\footnotetext{
' A good example of the failure to consider structural relationships is the inattention accorded balance sheet constraints by most producers or users of seasonally adjusted data.

The papers by Engle, Granger, Pierce, and Wallis, also included in this working paper, represent important building blocks in this process.
} 


\section{REFERENCES}

1. Kaufman, Herbert, and Lombra, Raymond. "ShortRun Variations in the Money Stock." Southern Economic Journal (April 1977): 1515-1527.

2. Lombra, Raymond, and Torto, Raymond. "The Strategy of Monetary Policy." Economic Review (September/October 1975): 3-14.

3. Nerlove, Marc. "Spectral Analysis of Seasonal Adjustment Porcedures." Econometrica 32 (July 1964): 241286.

4 Plosser, Charles I. "A Time Series Analysis of Seasonality in Econometric Models." Included in this report.

5. Sims, Christupher A. "Seasonality in Regression." Journal of the American Statistical Association 69 (September 1974): 618-626.

6. Stephenson, James, and Farr, Helen. "Seasonal Adjustment of Economic Data by Application of the General Linear Statistical Model." Journal of the
American Statistical Association 67 (March 1972): 3745.

7. U.S. Federal Reserve System, Board of Govemors of the Federal Reserve System. Improving the Monetary Aggregates, report by the Advisory Committee on Monetary Statistics. Washington, D.C.: Government Printing Office, 1976.

8. - Seasonal Adjustment of M1: Currently Published and Alternative Methods, by Edward R. Fry. Staff Economic Studies No. 87. Washington, D.C.: Government Printing Office, 1976.

9. - "Seasonal Adjustment of the Monetary Aggregates," by David A. Pierce and Neva VanPeski. In Improving the Monetary Aggregates: Staff Papers. Washington, D.C.: Government Printing Office, 1976.

10. Wallis, Kenneth F. "Seasonal Adjustment and Relations Between Variables." Journal of the American Statistical Association 69 (March 1974): 18-31. 


\title{
RESPONSE TO DISCUSSANTS
}

\author{
Charles I. Plosser \\ Stanford University
}

In his paper, Gregory Chow lists six characteristics of the general approach for the analysis of seasonal economic models that I have proposed. I would like to take this opportunity to briefly comment on these characteristics and clarify some of the issues involved.

Chow's first point seems to suggest that econometric models should be built to explain the seasonally adjusted data rather than the observed series. The notion that seasonally adjusted data are the only data of interest or relevance to the economist for the purpose of testing economic theories seems to stem, in part, I think, from the overused notion of breaking an economic time series up into trend-cycle, seasonal, and random components and assuming that the economic model exists solely between the trend-cycle components. As pointed out in the subsection on an approach to the analysis of seasonality in structural models of my paper, this approach could be used and would lead to very explicit restrictions on the form of the FE's and TF's. Alternatively, as in the approach suggested by Chow in his remarks, one can impose the restriction that the series can be decomposed into the sum of three unobserved components and obtain a seasonally adjusted series first, before the model is estimated. This seems to me to be a distinct disadvantage. It would be preferable to set up hypotheses to be tested regarding the decomposition rather than assume. it takes a certain form and then never bother to investigate the validity of the assumption.

There is another more fundamental reason for not proceeding in this manner where it is assumed that the appropriate economic model exists exclusively between the so-called cyclical components. As discussed briefly at the end of my paper, the role of anticipations has played an increasingly important function in economic theory, since the permanent income hypothesis was advanced by Milton Friedman. In models in which expectations play an important role, the appropriate distinction to be made is between anticipated and unanticipated phenomenon and not some arbitrarily chosen decomposition dealing with trends, cycles, and seasonal components. In making the distinction between anticipated and unanticipated effects, it would seem appropriate to merely consider seasonal fluctuations as a contribution, primarily, to the anticipated component. If such a model is appropriate, the economist or econometrician should be considering a different type of adjustment procedure (e.g., a prewhitening filter that reduces a series to white noise), rather than one that focuses only on the seasonal component.

The second and third points listed by Chow refer more to the specific example I considered rather than the general approach. The modelbuilder certainly has the option to allow structural parameters (elements of $\mathrm{H}_{11}$ or $\mathrm{H}_{12}$ ) to vary seasonally as well as to model the exogenous variables in a different manner. Both of these approaches would have implications for the forms of the TF's and FE's that could be checked against the data.

The fourth point raised by Chow is that the algebra is very complicated, and, therefore, the approach "might be applicable only to very small and very specialized models." Clearly, more experience is needed in applying the approach suggested here, but it is not necessarily true that only small models can be considered. Many of the characteristics that cause the algebraic manipulations to be simplified are actually found in many of the larger econometric models, including either a fully recursive or a block recursive structure. Consequently, larger models would not necessarily be more complicated to analyze.

The fifth characteristic noted by Chow concerns the role of expectations that was previously discussed. The sixth point asserts that the procedures do not yield a decomposition into the usual set of unobserved components. In light of this discussion, it is not clear whether such a decomposition is desirable or not. However, analysis of the sort suggested can be helpful in understanding the types of adjustment procedures that might be appropriate. The FE's, e.g., are in the form of univariate time series models, and there have been various methods suggested for decomposing these models if such an adjustment is of interest.

I hope that this discussion clarifies some of the issues in question. Although our techniques may differ, it is clear that Chow and I both favor incorporating seasonality into econometric models and feel that there may be much to leam from doing so. 


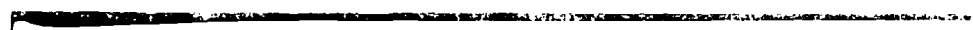




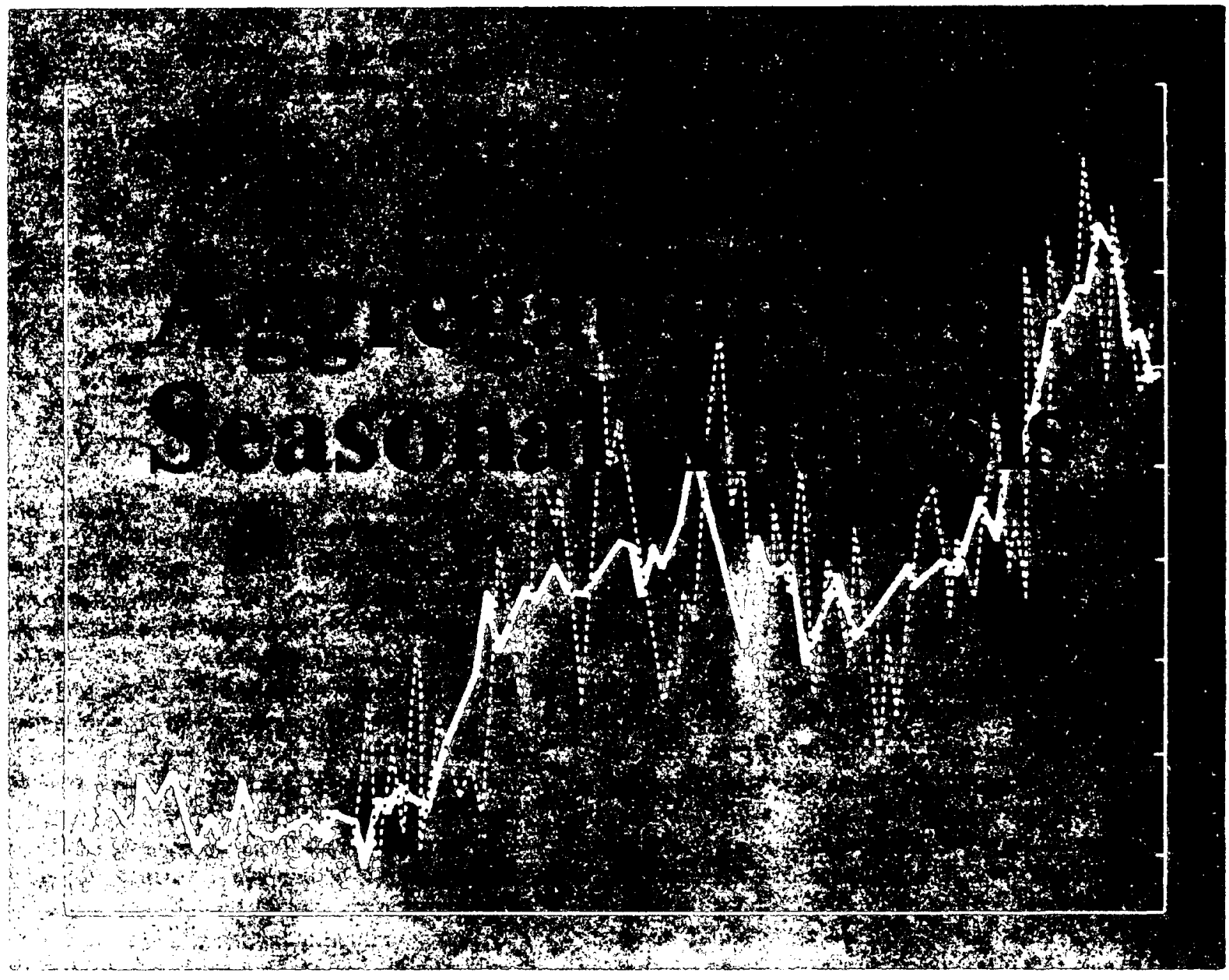

